https://helda.helsinki.fi

Cooperation in Games and Epistemic Readings of Independence-Friendly Sentences

Barbero, Fausto

2017-09

Barbero , F 2017 , ' Cooperation in Games and Epistemic Readings of

Independence-Friendly Sentences ' , Journal of Logic, Language and Information , vol. 26 , no. 3 , pp. 221-260 . https://doi.org/10.1007/s10849-017-9255-1

http://hdl.handle.net/10138/236594

https://doi.org/10.1007/s10849-017-9255-1

cc_by

acceptedVersion

Downloaded from Helda, University of Helsinki institutional repository.

This is an electronic reprint of the original article.

This reprint may differ from the original in pagination and typographic detail.

Please cite the original version. 


\title{
Cooperation in games and epistemic readings of Independence-Friendly sentences
}

\section{Fausto Barbero}

\begin{abstract}
In the literature on logics of imperfect information it is often stated, incorrectly, that the Game-Theoretical Semantics of Independence-Friendly $(I F)$ quantifiers captures the idea that the players of semantical games are forced to make some moves without knowledge of the moves of other players. We survey here the alternative semantics for $I F$ logic that have been suggested in order to enforce this "epistemic reading" of sentences. We introduce some new proposals, and a more general logical language which distinguishes between "independence from actions" and "independence from strategies". New semantics for $I F$ logic can be obtained by choosing embeddings of the set of $I F$ sentences into this larger language. We compare all the semantics proposed and their purported game-theoretical justifications, and disprove a few claims that have been made in the literature.
\end{abstract}

Keywords Independence-Friendly logic $\cdot$ Subgame Semantics · coordination · imperfect recall $\cdot$ subjective strategies $\cdot$ dominance solvability

Independence-Friendly Logic is an extension of first-order logic which allows expressing independence among quantifiers. Synctactically, this is specified by "slashed quantifiers" of the form $(\exists v / V)$, where $V$ is a finite set of variables, with the intended reading "there is a $v$ independent of the variables in $V$ ". Such languages were introduced in a form close to the modern one in Hintikka and Sandu (1989) (with some anticipations in Humberstone (1987), where a similar device was suggested in order to attempt a compositional analysis of the Henkin quantifiers of Henkin (1961)). In that paper it is suggested that these languages may be given a semantics by means of a generalization of the Game-Theoretical Semantics (GTS) of first-order logic; a first

Fausto Barbero

University of Helsinki, Department of Philosophy, History, Culture and Art Studies, P.O. Box 24, Unioninkatu 40 A, FIN - 00014, Finland, Tel: +358 2941 911, Fax: +358 294128060 E-mail: fausto.barbero@helsinki.fi 
detailed account of this approach can be found in Sandu (1993). In the same way as the truth of a first-order sentence in a structure can be characterized as the existence of a winning strategy in a specific 2-player semantic game of perfect information, appropriate games of imperfect information yield a definition of truth (and falsity) for $I F$ logic. Both kinds of games are played in the same way. The two players, usually called Verifier (or ᄏloise) and Falsifier ( $\forall$ belard) examine the synctactical tree of sentence $\varphi$ starting from the most external logical operator. One path in the synctactical tree is followed, and an assignment $s$ is gradually constructed ( $\forall$ belard picks an element from $M-$ the structure under consideration - every time a universal quantifier is met, while $\exists$ loise takes care of existential quantifiers); when a literal $\alpha$ is reached, victory is assigned to either player depending on whether $s$ satisfies $\alpha$ or not. Now the difference between the first-order and the $I F$ case lies not in the rules of the games, but instead in the truth conditions: a first-order sentence $\varphi$ is said to be true in $M$ if $\exists$ loise has a winning strategy in the corresponding game; but if $\varphi$ is an $I F$ sentence, truth is postulated to be equivalent to the existence of a winning strategy of a special kind (a uniform winning strategy). A uniform strategy makes choices for quantifiers of the form $(\exists v / V)$ without making use of parameters associated to the variables in the slash set $V$; this restriction encodes the game-theoretical requirement of imperfect information. See section 2 below for a more detailed description of the games and the standard semantics of $I F$ logic.

The resulting semantics is a conservative extension of first-order semantics, once $I F$ quantifiers of the form $(Q v / \emptyset)$ are identified with first-order quantifiers $Q v$. Yet with this semantics come some worries; first of all, the fact that open formulas do not get a meaning from it ${ }^{1}$. Secondly, the possible indeterminacy of the semantical game, which implies that a sentence might be neither true nor false, in case neither $\exists$ loise nor $\forall$ belard has a winning strategy; this is exemplified by the game $G(\varphi, M)$, with $\varphi:=\forall x(\exists y /\{x\}) x=y$ and $M=$ $\{a, b\}$, shown in Figure 1. Here the dotted line connects two nodes (histories) that are indistinguishable for the player who is supposed to make a move at the end of such history (in this case, ᄏloise). Clearly, none of the players has a uniform winning strategy, and $\varphi$ is undetermined on $M$.

Thirdly, with Game-Theoretical Semantics of imperfect information, new unexpected phenomena like signalling ${ }^{2}$ emerged; we shall say more about this later.

In parallel with the formal presentation of GTS in terms of game theory, the literature on $I F$ logic has been littered with informal explanations of GTS

\footnotetext{
1 Of course compositionality can be restored, but a price is to be paid; the usual notion of "being satisfied by an assignment" is inadequate to express the meaning of open formulas (Hodges (1997), Cameron and Hodges (2001)).

2 The presence of signalling phenomena reveals that $I F$ logic does not only allow partially ordered quantification; it also allows dependency between quantifiers to be an intransitive relation. Hintikka (1996) attempted to eliminate this feature by means of the implicit assumption that quantifiers of the same type $(\exists$ or $\forall)$ be always independent of each other. This stipulation, however, has more unpleasant consequences; with it, $I F$ logic is not anymore a conservative extension of first-order logic (see Janssen (2002)).
} 


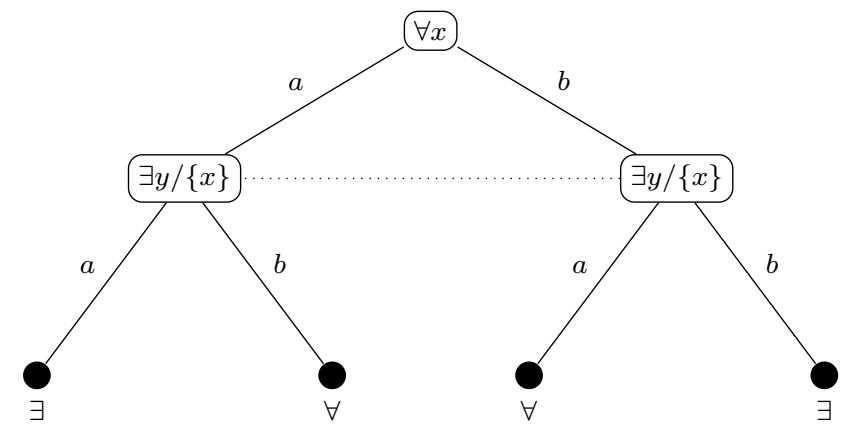

Fig. 1 The semantic game for $\forall x(\exists y /\{x\})(x=y)$ in a structure $M=\{a, b\}$

with an epistemic flavour. Think of $\exists$ loise and $\forall$ belard as two teams of players (one player for each quantifier or connective occurring in the sentence under consideration). Then, the quantifier $(\exists v / V)$ is often quoted as meaning "a value for $v$ can be chosen (by the corresponding player) without knowledge of the values assigned to $V$ ". This seemingly innocuous periphrasis turned out to be wrong, that is, not to correspond to the actual meaning of $I F$ sentences as induced by Game-Theoretical Semantics. It was first observed in Janssen (2001) that the sentence $\exists x(\exists y /\{x\})(x=y)$ is valid according to GTS (indeed, "choosing $a$ for both $x$ and $y$ " is a uniform winning strategy for $\exists$ loise, for any fixed element $a$ of the model), but should be non-true (in structures with at least two elements) under the epistemic reading, since it asserts that two distinct players have the ability to pick one and the same element from a structure without knowledge of the other player's choice. The seemingly counterintuitive truth value assigned by GTS comes from the assumption (implicit in the usual modelization of game theory) that players can coordinate their strategies, and so for example decide a priori that they will both choose a fixed element $a$. In Barbero (2013), the author considered more examples of these kinds of discrepancies and attempted a classification of them.

Understanding the distinction between GTS and the epistemic reading is important in order to make sense of many of the claims that have been put forward in the literature. For example, Hintikka (2013) interestingly points out that contingent statements like "there is someone such that if she loses money in the stock market next year, everyone will do so" are not correctly captured by what may seem to be their first-order translation: $\exists x(A(x) \rightarrow \forall y A(y))$ is indeed a validity. He then asserts that the classical implication must be replaced with a slashed one, $\rightarrow x^{3}$, in order to obtain the correct reading. But, under the GTS semantics, sentence $\exists x(A(x) \rightarrow / x \forall y A(y))$ is equivalent to $\exists x(A(x) \rightarrow \forall y A(y))$; Hintikka's claims make sense only if we assume that he had the epistemic reading in mind.

3 A formula of the form $\psi \rightarrow / x \chi$ is presumably interpreted by Hintikka as an abbreviation for $\neg \psi \vee /\{x\} \chi$; the (GTS) semantics of $\vee_{/\{x\}}$ is defined analogously to that of $(\exists v /\{x\})$. 
A few publications, starting with Janssen's Subgame Semantics papers (Janssen (2001), Janssen (2002)), have been advancing proposals on how to define a semantics for $I F$ sentences which respects the epistemic intuitions. The proposals that have been made are numerous, variegated and in general rather inconclusive and insufficiently justified. Some are grounded on gametheoretical concepts, while others on more ad hoc arguments. Claims have been made for some of the game-theoretical solutions to coincide with some of the ad hoc ones, and so to justify them; but we do not know of any proof of such statements. The main purpose of the present paper, which is a shortened and largely amended version of section 4 of the author's PhD thesis, Barbero (2014), is to collect together these ideas, compare them and make some order. We also introduce some new proposals, mostly of game-theoretical nature, and a generalization of $I F$ languages which allows discussing separately the notions of "knowledge of players' choices" and of "knowledge of players' strategies".

In section 1 we briefly outline the general framework in which we compare different logics. In section 2 we review the standard semantics of $I F$ logic, and then (section 3) we see in more detail how it clashes with the epistemic reading, and we give a sketch of what characteristics should be required for a good semantics of the epistemic reading. Section 4 looks at the notion of subjective strategy as a naive game-theoretical explanation of the (first version of) Subgame Semantics; sections 5 and 6 examine the two different versions that have been proposed of Subgame Semantics. Section 7 considers three different proposals that have been made by Sevenster (Sevenster (2007)) and Janssen (Janssen (2005), Janssen (2007)) to redefine the semantics of $I F$ logic (and perhaps to give a grounding to Subgame Semantics) in terms of gametheoretical notions of weak domination of strategies and rationalizability. In Section 8 we consider a notion of iterated elimination of weakly dominated strategies known as dominance solvability. In section 9 we introduce the $C S$ languages, which distinguish between "independence from choices" and "independence from strategies"; and assign them a game-theoretical semantics (Cartesian Semantics) based on the idea that players know that the other players are rational, but they cannot freely communicate with each other. We also propose some embeddings which allow defining new readings of $I F$ sentences by means of Cartesian Semantics. In section 10 we consider briefly the Independence logic of Grädel and Väänänen (2013), which has some deceiving similarity with Cartesian Semantics. Finally, in section 11 we compare the most promising systems by means of a number of revealing examples. These show that most of the readings of $I F$ languages considered do not coincide; in particular, we disprove the claim of Janssen (2005), Janssen (2007) that Rationalizability Semantics might be the correct game-theoretical justification of Subgame Semantics on finite structures. 


\section{General framework}

In the following we shall compare different semantics for one same syntax, or, in more complicated cases, logics with mutually different syntactical structure. We write $M \models{ }_{L} \varphi$ to say that, according to logic $L$, sentence $\varphi$ is true in structure $M$ (specific semantical clauses for truth will be given for each of the logical systems considered in the paper). We say that a class $K$ of structures is defined by $\varphi$ according to $L$ if, first of all, $\varphi$ is a sentence of $L$; and secondly, if for all structures $M$,

$$
M \in K \Longleftrightarrow M \models_{L} \varphi .
$$

We then say that a class of structures $K$ is definable in $L$ if there is a sentence $\varphi$ of $L$ which defines $K$ according to $L$.

The various logics will be differentiated according to two conceptual criteria: comparison of expressive power, and inclusion of "sets of truths". More precisely, if we are given two logics $L_{1}$ and $L_{2}$, we write 1) $L_{1} \leq L_{2}$ to mean that every class of structures definable by a sentence of $L_{1}$ is also definable by a sentence of $L_{2}$, and 2) $L_{1} \subseteq L_{2}$ to mean that, on all structures $M, M \models_{L_{1}} \varphi$ implies $M \models_{L_{2}} \varphi$. When the relation $\leq$, resp. $\subseteq$, holds in both directions, we use the symbols $\equiv$, resp. $=$. We also write $\leq_{\text {fin }}, \subseteq_{\text {fin }}$ for the analogous notions restricted to finite structures.

The relationship $L_{1} \subseteq L_{2}$ is meaningful only in case the logics $L_{1}$ and $L_{2}$ share the same syntax. When this is not the case, the relationship $L_{1} \subseteq L_{2}$ will be intended as the existence of an (injective, but not necessarily surjective) truth-preserving translation $\tau$ of $L_{1}$ sentences into $L_{2}$ sentences; that is, a function such that, for any structure $M$ and any sentence $\varphi$ of $L_{1}$,

$$
M \models{ }_{L_{1}} \varphi \Longleftrightarrow M \models_{L_{2}} \tau(\varphi) .
$$

\section{The standard semantics}

As a reference for the reader, we review in some detail the standard semantics of (first-order and) IF logic (Mann et al. (2011)).

Consider for the moment a first-order syntax which allows the use of constant, function and relation symbols, and of logical constants $\forall v, \exists v, \wedge, \vee, \neg$, with the obvious formation rules; we may assume, for simplicity, that formulas be in negation normal form (with negations occurring only in front of atomic formulas). One can associate, to every pair $(\varphi, M)$ consisting of a sentence and a structure, a semantic game $G(\varphi, M)$; more generally, it is technically convenient to allow $\varphi$ to be any formula (possibly with free variables) and to take into consideration, as a further parameter, an assignment $s$, defined at least on all the free variables of $\varphi$; then, one can speak of games $G(\varphi, M, s)$ and consider $G(\varphi, M)$ to be an abbreviation for $G(\varphi, M, \emptyset)$.

Let us fix $\varphi, M$ and $s$. The corresponding game $G(\varphi, M, s)$ has two players, usually called $\exists$ loise (or Verifier) and $\forall$ belard (or Falsifier). The game follows 
the synctactical tree of the sentence, starting from the root; each logical constant prompts a move. If $\forall v$ is met (that is, we are playing $G(\forall v \psi, M, s)$ for some formula $\psi$, structure $M$ and assignment $s$ ), then $\forall$ belard chooses from $M$ a value $a$ for $v$, and the game $G(\psi, M, s(a / v))$ associated to the immediate subformula $\psi$ is played ${ }^{4}$; an analogous move of $\exists$ loise is triggered by $\exists v$. A conjunction corresponds to the choice of a conjunct by $\forall$ belard, and the game proceeds in the corresponding subtree (say, in a game $G(\psi \wedge \chi, M, s)$ $\forall$ belard decides whether it is the case to proceed and play $G(\psi, M, s)$ or instead $G(\chi, M, s))$. Disjunctions are similarly treated by $\exists$ loise. When a literal $\alpha$ is reached (say, $G(\alpha, M, s)$ is played), one checks whether $\alpha$ is satisfied by $s$ in $M$ (that is, satisfied by the values that have been picked during the game); if it is, $\exists$ loise wins, otherwise $\forall$ belard wins. Figure 2 shows a representation of the game tree of the game $G(\forall x \exists y(x=y), M, \emptyset)$, for $M=\{a, b\}$.

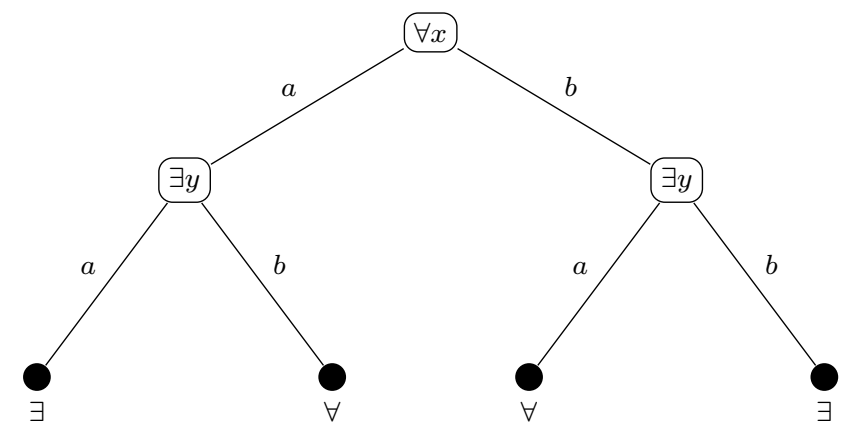

Fig. 2 The semantic game for $\forall x \exists y(x=y)$ in a structure $M=\{a, b\}$

Each node in the tree represents a possible history of the game; edges list the legal moves that can be performed after a given node (history) is reached. The symbols $\forall, \exists$ drawn in the terminal (maximal) histories specify which player wins; the symbols in the non-terminal nodes specify what kind of move is going to be played next.

A strategy for a game of this kind is a function that associates to each history of the game a legal move; it is winning for a player $P$ if, following it, $P$ wins the game, whatever moves may the opponent choose. The truth of $\varphi$ in $M$ can then be defined as $\exists$ loise having a winning strategy in $G(\varphi, M)$, falsity as $\forall$ belard having one. One obtains exactly the same truth values of sentences that would come from Tarskian semantics. In the example in Figure 2 , the sentence is indeed true in $M$, thanks to $\exists$ loise's strategy "choose for $y$ the same value that $\forall$ belard chose for $x "$.

In the case of $I F$ logic, quantifiers are of the forms $(\forall v / V),(\exists v / V)$, for finite sets $V$ of variables (slash sets). The first-order quantifiers $\forall v, \exists v$ are identified, respectively, with $(\forall v / \emptyset)$ and $(\exists v / \emptyset)$. In the following, we show how

4 Of course here $s(a / v)$ denotes the assignment which assigns $a$ to $v$, and $s(w)$ to any variable $w \in \operatorname{dom}(s) \backslash\{v\}$. 
the new quantifiers are standardly treated by introducing imperfect information in the games. Following the definitions above, we see that at the end of each history $h$ of the game some assignment $s_{h}$ is being considered; we say $s_{h}$ is the assignment associated to $h$. Now suppose that two histories $h, h^{\prime}$ both end up in a choice for (one and the same occurrence of) $(\exists v / V)$; then, we say that $s_{h} \sim_{V} s_{h^{\prime}}\left(s_{h}\right.$ is indistinguishable from $s_{h^{\prime}}$ outside of $V$ ) in case $s_{h}(x)=s_{h^{\prime}}(x)$ for any variable $x \in \operatorname{dom}\left(s_{h}\right) \backslash V$. The corresponding histories will also be said to be indistinguishable modulo $V\left(h \sim_{V} h^{\prime}\right)$. Histories equivalent under this equivalence relation are said to form an information set; if an history $h$ is not associated to a quantifier choice, then $\{h\}$ alone is an information set. We can then say that a strategy $\sigma$ of $\exists$ loise is uniform if, whenever $h \sim_{V} h^{\prime}, \sigma(h)=\sigma\left(h^{\prime}\right)$. That is, the fact that $\exists$ loise is supposed not to know the values associated to $v$ while making a choice for $(\exists v / V)$ is encoded by the restriction that her strategies assign the same value to histories that are indistinguishable modulo $V$ (we will discuss later under what circumstances this last clause may fall short of an adequate characterization of the knowledge of $\exists$ loise). Dual definitions can be given for $\forall$ belard. We then say that an $I F$ sentence $\varphi$ is true if $\exists$ loise has a uniform winning strategy in $G(\varphi, M)$; false if $\forall$ belard has one.

In order to be able to discuss examples with more ease, we introduce some auxiliary concepts and notations. Given a strategy $\sigma$ of $\exists$ loise, we can decompose it in the following way: if we have an occurrence of a logical operator in $\varphi$, for example $(\exists y / Y)$, we may consider the set $H((\exists y / Y))$ of the histories of $G(\varphi, M)$ that end in that occurrence of $(\exists y / Y)$; the restriction $\sigma_{\uparrow H((\exists y / Y))}$, that we shall denote for example as $f_{y}, f_{\exists y}$ or $f_{\exists y / Y}$ (trying to avoid ambiguity), is called a strategy function associated to (the occurrence of) $(\exists y / Y)$. With some abuse of notation, we will write, e.g., $f_{y}(\hat{x}, \hat{z})$ (in case $(\exists y / Y)$ occurs under the scope of quantifiers on $x$ and $z$, and possibly of quantifiers over variables in $Y$ ) to denote the value that $\exists$ loise picks if she follows strategy function $f_{y}$ when values $\hat{x}$, resp. $\hat{z}$ have been chosen for $x$, resp. $z$. Similar conventions apply to $\forall$ belard. These conventions make it easier to speak of $\exists$ loise and $\forall$ belard as two teams of players (one individual player for each logical constant, except for negations).

\section{Standard versus epistemic semantics}

As we mentioned above, the seemingly innocuous liberalization of semantics to games of imperfect information has remarkable effects: for example, it enforces the introduction of undetermined semantic games, and thus a three-valued semantics; and it turns first-order logic into a system which is as expressive as existential second-order logic. It also allows the possibility of intransitive dependence between quantifiers, or signalling (Hodges (1997)), as for example in the sentence

$$
\forall x \exists y(\exists z /\{x\})(x+y=z)
$$


The structure of the sentence makes so that $y$ depends on $x$, and $z$ depends on $y$, but $z$ does not depend on $x$ (in this sense the relation of dependence is intransitive). Yet, observe that this sentence is true in $\mathbb{N}$, because the $\exists$ loise team, which takes care of choosing witnesses for the existential quantifications, may win the game by answering to any choice of value for $x$ with the strategy functions $f_{y}(x):=x, f_{z}(y):=2 y$. Here the statement " $/\{x\}$ " means that the player must make his choice without using the value of $x$, and indeed $x$ is not among the parameters of $f_{z}$; yet, a correct answer to $x$ is reconstructed from the value of $y$. The player associated to $\exists y$ is said to signal the value of $x$ to the player associated to $\exists z$. A well-known and more extreme example is the sentence $\forall x \exists y(\exists z /\{x\}) x=z$, which turns out to be valid thanks to the quantification over $y$. Notice that $y$ does not occur in the atomic part of the sentence, and so it would be a dummy variable in first-order logic; yet it plays a crucial role in the semantics of this $I F$ sentence. This difference is expressed by saying that first-order logic is local, while $I F$ logic is nonlocal. Sometimes in the literature also the phenomenon of nonlocality is referred to as "signalling"; we prefer to keep the two notions distinguished. With our terminology, there are logics which are local and yet have signalling patterns (e.g., Dependence logic and Dependence-Friendly logic, see Väänänen $\left.(2007)^{5}\right)$.

The policies about signalling are a particular instance of a more general problem: what forms of cooperation between players should be allowed in the semantical games? (Notice indeed that in the previous example the existential players are required to coordinate their strategies so that the signal is correctly decoded). The question has been rarely addressed at this level of generality; yet it appears in the literature, beginning with Janssen (2001). In the present paper we will examine some non-standard semantics for $I F$ languages, some of which appeared in the literature, while some are new, that are meant to either inhibit cooperation among players, or, otherwise, to allow greater control over forms of cooperation. In a sense, these logics are meant to express more faithfully the epistemic intuitions that usually come together with informal presentations of $I F$ logic; in particular, we want to take more seriously the presentation of $I F$ semantics as a game between two teams.

We return here to the remarkable (but rather special) example analyzed in Janssen (2001): sentence

$$
\exists x(\exists y /\{x\})(x=y) .
$$

According to standard $I F$ semantics, formula (1) is valid. Let us review the reason behind that. Given any structure, we can define a winning strategy for the $\exists$ loise team, like this:

\footnotetext{
5 Patterns equivalent to signalling sentences can be obtained in Dependence logic by means of peculiar combinations of dependence atoms and quantifiers, for example in sentences of the form $\forall x \exists z(=(x, z) \wedge \exists y(=(z, y) \wedge \psi)$; and in Dependence-Friendly logic, by means of quantifier sequences, e.g. the prefix $\forall x(\exists z \backslash x)(\exists y \backslash z)$. These kinds of patterns allow expressing concepts beyond first-order, such as infinity over any signature (Enderton (1970)) and NP-complete problems (Sevenster (2014)); actually, any concept definable in existential second-order logic can be expressed by signalling sentences (Barbero et al. (2017)).
} 
- Fix an element $a$ of the structure

- As a value for $x$, choose $a$

- As a value for $y$, choose $a$.

That is, the two $\exists$ loise players play according to the constant strategy functions $f_{x}()=a$ and $f_{y}()=a$. This strategy employs only 0 -ary functions and so it satisfies, trivially, any independence constraint. So, it seems that our restriction to uniform strategies does not force player $\exists y$ to play in ignorance of the value chosen by player $\exists x$. It seems that she can play "as if she knew that player $\exists x$ plays according to the constant strategy $a$ ". So, she manages indirectly to acquire knowledge of the value of $x$.

The nonstandard semantics we will consider all try to enforce the following:

We want formula (1) to be true in one-element domains, non-true elsewhere.

This is a very particular constraint, and it does not provide us with any mechanichal process to redefine the semantics of all $I F$ sentences. Very different suggestions have been and will be made, with very different justifications. The common underlying idea will be that of requiring, for truth, not only the existence of a winning strategy for the Verifiers; rather, the existence will be required of a winning strategy which is, in some sense, really playable when the players are forbidden to cooperate, or limited therein.

A more general guiding principle for devising and testing these kinds of semantics could be given by the notion of relevance that was introduced in Barbero (2013). Call an occurrence of a variable $x$ in the slash set $Y$ of $(\exists y / Y)$ a declaration of independence of $y$ from $x$; given an $I F$ quantifier prefix $\mathbf{Q}$ which contains exactly one declaration of independence of $y$ from $x$, call $\mathbf{Q}^{y \leftarrow x}$ the quantifier prefix which differs from $\mathbf{Q}$ in that $(\exists y / Y)$ is replaced by $(\exists y /(Y \backslash\{x\}))$. Then, we say that the declaration of independence of $y$ from $x$ occurring in $\mathbf{Q}$ is relevant if there are an $I F$ formula $\psi$ and a structure $M$ such that $\mathbf{Q} \psi$ and $\mathbf{Q}^{y \leftarrow x} \psi$ are sentences which have different truth values on $M$.

It would then seem natural to require that

We want all declarations of independence to be relevant.

But we do not know if this constraint is realistic or achievable with some sensible semantics ${ }^{6}$.

We want to make one more observation about example (1). In formula (1), information sets are, in a sense, violated. The second stage of the game is constituted by a set of indistinguishable nodes, one for each element of the structure; yet, each one of them is characterizable as "the node which is reached when $\exists x$ plays the costant function $a$ ", for a certain $a \in M$. Thus, knowledge

\footnotetext{
6 For sure, it would not be sensible to require the same constraint over the notion of sentence-relevance (also introduced in Barbero (2013)), which is analogous to relevance, but affects individual sentences instead of quantifier prefixes. For example, we do not want the declaration of independence in $\exists x(\exists y /\{x\})(x=y \vee x \neq y)$ to affect the truth value of what looks like a validity even under an (intuitive) epistemic reading.
} 
of strategies makes so that information sets do not represent anymore indistinguishability of nodes. This amounts not to an error in the modelization of $I F$ games; it is instead a common feature of games of imperfect recall ${ }^{7}$; see for example Halpern (1996). On some standard Game Theory texts this is taken as a definition of imperfect recall (Osborne and Rubinstein (1994), Definition 203.3). Notice here that there are two kinds of information being involved in our semantic evaluation of sentences:

a) Information (= direct access) about the values chosen by other players, in each single play, for variables.

b) Information about the strategy function chosen by each player ${ }^{8}$.

In $I F$ semantics, a) is administered according to information states, and it has a direct correspondence with syntax. About b), the GTS games are played as if each player knew all the strategy functions employed by his comrades, and none of the strategies employed by members of the rival team. The alternative semantics considered in this paper are such that information of type a) is treated as usual, while information of type b) is not allowed to flow as freely. As extremal cases, we may informally point out:

- Strict game-theoretical interpretations: each player does not know the strategy functions used by any other player; so, he cannot use them in order to define his own strategy function. It is to be expected that most forms of signalling are blocked.

- Lax game-theoretical interpretations: also access to information of type b) is regulated by the slash declarations; each player only knows the strategy functions of those comrades whose variable does not occur in the slash set. We can then expect that, while coordination of strategies is limited, signalling of values is still permitted.

- Very lax game-theoretical interpretations: information of type b) (about comrades) is always accessible.

We can also decide, in order to allow for maximum freedom, to employ two different slashing devices for information of kind a) or b). For example, we could write $\left(\exists z /\{x, w\}_{-}\{x, y\}\right)$ to express the fact that a strategy function $f_{z}$ for $z$ must be chosen as a $\{x, w\}$-uniform function and be defined without using strategy functions $f_{x}, f_{y}$.

We shall call $C S$ the set of formulas with such syntax (having an underscore set, and not only a slash set), and the corresponding logic. In section 9 we will design our semantics for this syntax. We shall then obtain a truth semantics $I F_{C S}$ for $I F$ logic as a special case of $C S$, by identifying each $I F$ quantifier

$$
(\exists v / V)
$$

7 A game has imperfect recall if some of the players in it can forget some of their previous moves or knowledge. In a sense, most $I F$ games are of imperfect recall (see Mann et al. (2011), Theorem 6.23).

8 This statement must be intended in its de dicto reading: we are talking about knowledge of which strategy function is chosen, not knowledge of the strategy as an object. 
with the $C S$ quantifier

$$
\left(\exists v / V_{-} V^{\exists}\right)
$$

where $V^{\exists}$ is $V$ restricted to existentially quantified variables. This means that each player of $\exists$ loise's team can see the strategies of exactly those comrades whose chosen values she can see. Otherwise stated, information of types a) and b) coincide for team comrades; it will be unimportant, due to our definition of the semantics of $C S$, to specify that $\exists$ loise players cannot see any of the strategies of $\forall$ belard players. This is a "lax" semantics according to our previous classification.

Standard $I F$ semantics is obtained when we identify each $I F$ quantifier $(Q v / V)$ with the $C S$ quantifier $\left(Q v / V_{-} \emptyset\right)$. This is an example of "very lax" semantics.

An example of "strong" semantics is obtained by identifying each $I F$ quantifier $(Q v / V)$ with the $C S$ quantifier $\left(Q v / V_{-} W\right)$, where $W$ is the set of all variables which are existentially quantified in quantifications which are superordinated to $(Q v / V)$. IF logic equipped with this semantics will be denoted as $I F_{0}$.

\section{Subjective strategies}

Janssen (2002) introduced a new semantics for $I F$ languages, which was called Subgame Semantics; this semantics is defined on $I F$ sentences, but the values it assigns to some couples (sentence, structure) are different from those which are generated by standard $I F$ semantics. Janssen himself admitted that the development of this semantics was quite an empiric enterprise. It seems that this search was driven partially by the attempt to give an intuitively sound interpretation to some specific examples, and partially by the attempt to fulfill some general abstract requirements. Before presenting Subgame Semantics (in the following sections) we want here to analyze these theoretical demands, and to propose a game-theoretical semantics which seems to satisfy them. This will help understand better where the peculiaritiess of Subgame Semantics come from.

Subgame Semantics was born as an answer to:

(A) a GAME-THEORETICAL concern: Janssen wanted all IF sentences to represent the behaviour of players of games without communication. The players should know the moves that are not in their slash sets, and be ignorant about the slashed moves; they should not be able to reconstruct the slashed information by different means (signalling, coordination, etc.). In this way, a formula like $\exists x(\exists y /\{x\})(x=y)$ should be non-true in every domain with at least two elements.

(B) a MATHEMATICAL concern: declarations of independence between quantifications should express functional independence. So, if a sentence declares $y$ to be independent from $x$, and the game is won by reaching a final atomic formula $\varphi$ and an assignment $s$, then the game should be won 
also in $(\varphi, s(a / x))$, for any $a$ in the model. This is not the case, under standard $I F$ semantics, for formula $\exists x(\exists y /\{x\})(x=y)$. (For reasons that will be explained later, this request will be weakened in the following way: asking only that $(\varphi, s(a / x))$ is a win for any $a$ such that there is a $b$ which makes $(\varphi, s(a / x, b / y))$ a win).

(C) a LOGICAL concern: the meaning of a subformula should be independent of the context in which the subformula appears (for example, it should not depend on variables which do not occur in the subformula itself; using the terminology introduced earlier, the logic should be $l o$ $\mathrm{cal}$ ). This is somewhat related to (A): dependence on the context allows forms of communication ("signalling") between the players, as in formula $\forall x \exists z(\exists y /\{x\})(x=y)$ ( $z$ does not occur in subformula $(\exists y /\{x\})(x=y)$, yet, in standard $I F$ logic it is essential in the evaluation of the sentence). Notice however that locality does not block all possible forms of communication (consider for example the sentence $\forall x \exists z(\exists y /\{x\})(x=y \wedge z=z)$ : here we cannot appeal to locality in order to establish that $\exists z$ is a dummy quantifier).

These requirements are of a very variegated nature, and finding a semantics which satisfies them all, without any guiding principle, looks rather difficult. We make here a first, coarse attempt to reduce these expectations to a semantics which is based on a simple game-theoretical solution concept. What we will choose is not the most obvious or well-known solution concept. It is rather a sort of ghost which haunts game-theoretical literature, and especially the literature on Alternating Time Logics: the notion of subjective strategy. To explain what it means for a strategy to be objectively winning, we quote from such literature (Jamroga and van der Hoek (2004)):

...for planning purposes, the agent should be rather interested in having a strategy and knowing it (i.e. not only knowing that he has some strategy)!

This relates with the concern (A) stated above: declarations of independence may happen to be irrelevant precisely because $\exists$ loise knows, at any stage of the game, which (global, objective) strategy she is applying. Instead, in an imperfect recall scenario it may be also reasonable that, in each history, she only knows the (local) strategy function that she is meant to use in the current node (and yet be sure that such choice is winning). In the example $\exists x(\exists y /\{x\})(x=y)$ (see Figure 3), ᄏloise's second strategic function is a constant choice (say, either $a$ or $b$ ). Suppose she is in this information state, and her global strategy is "choose $a$ for $x$, choose $a$ for $y$ ". It would make no sense to state that now she does not know that she is supposed to choose $a$ for $y$. But, does she know whether she has had to choose $a$ or $b$ for $x$ ? We can imagine both scenarios.

In one scenario she knows, and thus knows the whole strategy she is adopting. In her current state, the strategy function "choose $a$ for $x$ " is objectively a winning strategy; but $\exists$ loise also knows at this point that her str. function will lead to a win; therefore her strategy is also subjectively winning. 


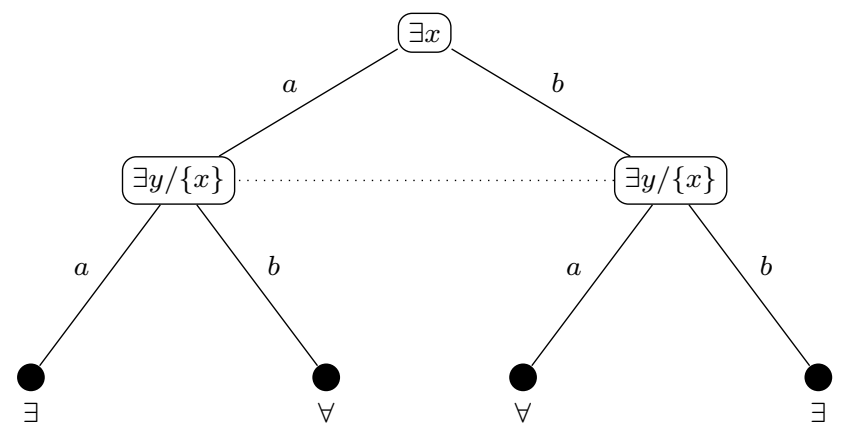

Fig. 3 The semantic game for $\exists x(\exists y /\{x\})(x=y)$ in a structure $M=\{a, b\}$

In the other scenario, $\exists$ loise has forgotten what she was meant to choose for $x$. So, in the moment when she is supposed to choose a value for $y$, she does not know whether she is in the history $h_{a}$, where $a$ has been chosen for $x$, or in history $h_{b}$, where $b$ has been chosen. Thus, even though she knows she must choose $a$ for $y$, she does not know whether this choice will lead to a victory. It will, if she is in $h_{a}$; it will not, if she is in $h_{b}$. So, her global strategy is objectively but not subjectively winning in the current information state. This phenomenon can be thought also in these terms: in games of imperfect recall, total or partial knowledge of one's own strategy may be represented as a constraint which shrinks (partitions) the information states; or, we may think that knowledge of the strategy forces us to consider a smaller game tree, in which only reachable nodes are represented. This process may reduce the size of information states in case the game is a game of imperfect recall.

We called these notions by the name of ghosts, because we never happened to find in the literature a formal definition. Thus, we will try to formulate it here. Notice first of all that the notion of "being subjectively winning" does not apply to classical game trees, which have only one root: it is of some interest only in case the game may start from different roots (according, for example, to some random choice procedure), and in case some of these initial nodes are in a common information state. To emphasize the fact that we are not considering classical games, we will speak of game forests whenever there is more than one initial node.

A history $h$ can be decomposed in consecutive segments, each of them constituted by some consecutive nodes. For example, we might split a history $h$ in three segments $p, q, r$ such that $p$ contains the root node and some of the nodes below; below that, a few nodes constitute $q$; the last nodes until the end of $h$ constitute $r$. In this case, we can write $h$ as a concatenation of $p, q, r$ : $h=p^{\frown} q \frown r$. With this notation, we can define subforests:

Definition 1 A subforest of a game forest $F$ is a set $G$ of segments of histories of $F$ satisfying the condition that, if $p^{\frown} q \frown r \in F$ and $q \in G$, then also $q \frown r \in G$. The information sets of $G$ are all the nonempty intersections $S \cap G$, where $S$ is an information set of $F$. 
Plainly speaking, a subforest $G_{C}$ is obtained by choosing a subset $C$ of a game forest, and keeping only those nodes and actions that occur in $C$ or after a node of $C$. We shall say that $G_{C}$ is the subforest generated by $C$.

Denote by $A(S)$ the set of actions that can be performed in any node of the information set $S$. As usual, we may think of a strategy, for a game forest, as a function $f$ from the set of information sets to the set of actions, such that for all information states $S, f(S) \in A(S)$ (which, in the case of $I F$ games, means that either $f(S) \in M$ or $f(S) \in\{L, R\}$, depending on whether the next move associated to $S$ is a quantifier or a connective move).

We are mainly interested here in subforests generated by an information set of an $I F$ game. This will make things fairly simpler.

Definition 2 Let $G(\varphi, M)$ be an $I F$ game, and let $S$ be one of its information sets. A function $f$ is a local subjectively winning strategy for the subforest $G_{S}$ if

1. It is the restriction to $G_{S}$ of a winning strategy of $G(\varphi, M)$

2. For each $s \in S, f$ restricted to $G_{\{s\}}$ is a winning strategy for (the game) $G_{\{s\}} \cdot$

Definition 3 A strategy $f$ for an $I F$ game $G=G(\varphi, M)$ is subjectively winning if, for each information set $S$ that can be reached by playing according to $f$, its restriction to $G_{S}$ is a local subjectively winning strategy in $G_{S}$.

Notice that, even though it is not immediately obvious from the statement, this definition enforces the local subjectivity condition on all information states that are crossed by histories which are indistinguishable from the actual one.

Definition 4 We say that $\varphi$ is true in $M$ according to Subjective Strategy Semantics, and we write

$$
M \models S S S \varphi
$$

if $\exists$ loise has a subjectively winning strategy in $G(\varphi, M)$.

Example 1 Let us go back to the sample sentence $\exists x(\exists y /\{x\})(x=y)$, in a structure $M=\{a, b\}$ (as depicted in Figure 3 ). Let us call $G$ the associated game. It has two information states: $S_{1}$, which contains the root node, and $S_{2}$, containing the two remaining nodes (say, $e_{a}$, where $a$ has been chosen for $x$, and $\left.e_{b}\right) . S_{2}$ is crossed by any play of the game. Yet, the strategy function "choose $a$ for $y$ " is not winning in $G_{\left\{e_{b}\right\}}$, and the strategy function "choose $b$ for $y$ " is not winning in $G_{\left\{e_{a}\right\}}$. So, there is no strategy satisfying condition 2. of the definition of local subjective winning strategy: $M \not \forall_{S S S} \exists x(\exists y /\{x\})(x=y)$.

Example 2 Let us now consider, over the same structure, Hodges' signalling formula $\forall x \exists y(\exists z /\{x\})(x=z)$. Let $S$ be the information state constituted by the two nodes circled in darker lines in Figure 4. Call $e_{1}$ its left node, $e_{2}$ its right node. 


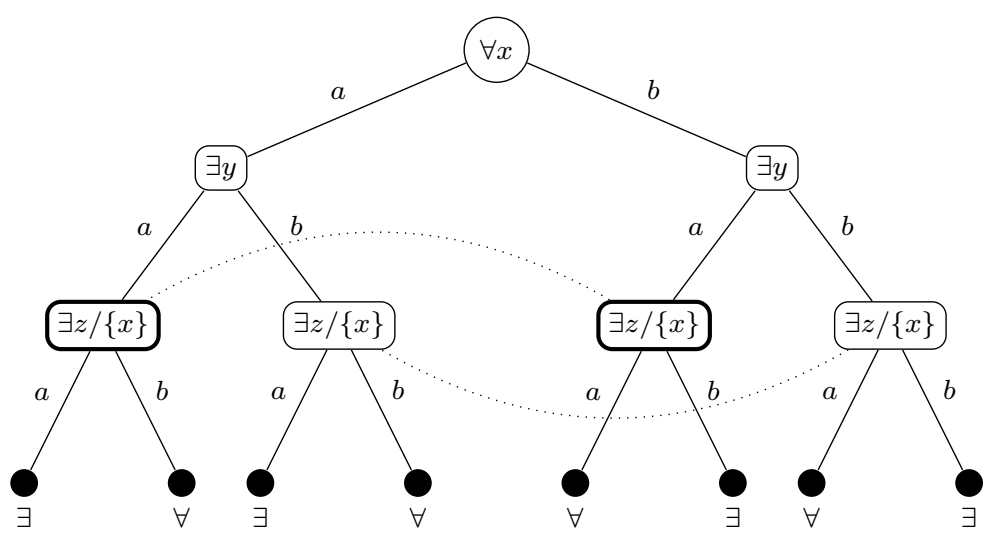

Fig. 4 The semantic game for $\forall x \exists y(\exists z /\{x\})(x=z)$ in $M=\{a, b\}$

There are two winning strategies for $\exists$ loise. The first is "choose for $y$ the value of $x$, choose for $z$ the value of $y$ "; call it $f$. But we can check that this is not a subjectively winning strategy. Indeed, state $S$ can be reached when $f$ is used (it happens in case $\forall$ belard chooses $a$ ). But the restriction of $f$ to $G_{\left\{e_{2}\right\}}$ is not a winning strategy for $G_{\left\{e_{2}\right\}}$.

The other winning strategy is "choose for $y$ the value that was not chosen for $x$, choose for $z$ the value that was not chosen for $y$ ". Again, $S$ can be reached following this strategy (in case $\forall$ belard chooses $b$ ). So this strategy is not subjectively winning, either, because it leads to a loss from the leftmost node $e_{1}$.

So, $M \forall_{S S S} \forall x \exists y(\exists z /\{x\})(x=z)$. We see that some form of signalling fails under Subjective Strategy Semantics. More precisely, this example describes a situation in which the second $\exists$ loise player sees the signal that is sent by the first $\exists$ loise player, but does not know how to interpret it (she does not know which strategy the other player is using for the purpose of communication).

Sadly, this semantics assigns very counterintuitive values to some sentences:

Example 3 The following example is taken from Janssen (2002), where it is attributed to J. Väänänen. Consider sentence $\exists x(\exists y /\{x\})(x=0)$, in a language containing at least the constant symbol 0 .

Any strategy $\sigma$ in which $\exists$ loise chooses $0^{M}$ for $x$ is winning. But for it to be subjectively winning, we need $\sigma_{y}$ (the strategy function of $\sigma$ which picks a value for $y$ ) to constitute a winning strategy also in the rightmost node, which cannot be the case. So, this sentence is non-true due to the dummy variable $y$. 


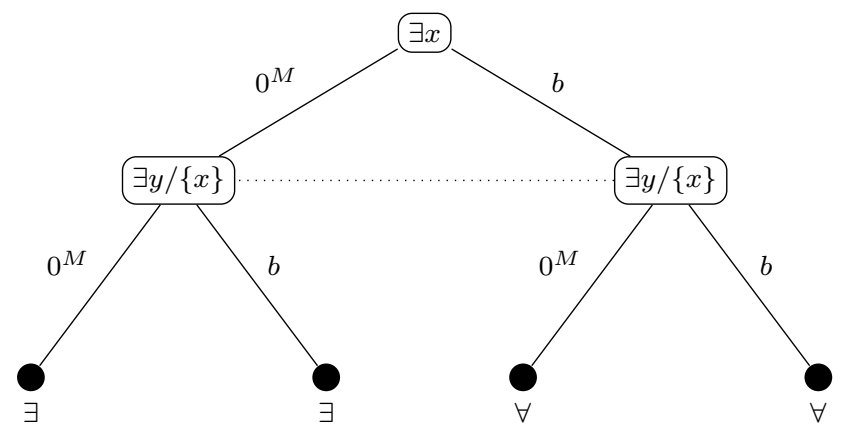

Fig. 5 The semantic game for $\exists x(\exists y /\{x\})(x=0)$ in a structure $M=\left\{0^{M}, b\right\}$

\section{Subgame Semantics, first version}

In the previous section we staged a mental experiment, in an endeavour to isolate the core idea of Subgame Semantics. Now it is time to show the real thing, with all of its contingent peculiarities. But we have a surprise: in the literature we find two different versions of Subgame Semantics! The two publications Janssen (2001) and Janssen (2002) may look at first as two versions of the same paper; a closer investigation reveals instead that the logical systems introduced in each work present many substantial differences. Here we introduce the oldest version, with some interpolation. We add explicit clauses for conjunction and universal quantification.

We consider here a variant of $I F$ syntax in which slashes may occur after existential quantifiers and disjunctions (that is, we may have subformulas of the form $(\exists v / V)$ or $(\vee / V)$, but not of the form $(\forall v / V)$ or $(\wedge / V))$. Janssen also states that only singletons should be considered as possible slash sets, but we do not see any clear necessity for this restriction.

The set of free variables of a formula is defined inductively as for first-order formulas, with the following special clauses:

$$
\begin{aligned}
& F V\left(\psi \vee_{/ W} \chi\right)=F V(\psi) \cup F V(\chi) \cup W \\
& F V((\exists y / W) \psi)=(F V(\psi)-\{y\}) \cup W .
\end{aligned}
$$

Definition 5 (Janssen (2001), 4.2; Janssen (2002), 10.3)

Given an $I F$ formula $\varphi$ and an assignment $s$ s.t. $F V(\varphi) \subseteq \operatorname{dom}(s)$, we write $s_{\varphi}$ for its restriction to the free variables of $\varphi$. Given a structure $M$, let $P_{\varphi}$ be the set of assignments on the set $F V(\varphi)$.

These definitions clearly go in the direction of enforcing the locality of $I F$ logic. In the same spirit, some small but very significant changes are made in the definition of the semantic games.

Definition 6 (Janssen (2001), 4.3; Janssen (2002), 10.4)

To each pair $(\varphi, M), \varphi$ being an $I F$ formula, $M$ a structure, we associate an SGS game $G^{S G S}(\varphi, M)$. The possible positions of the game are the 
assignments, ranging in $M$, to the free variables of $\varphi$ (that is, the elements of $P_{\varphi}$ ). The game allows the following moves (assuming that the game is played from a position $s$ ):

- If $\varphi$ is $R\left(x_{1}, \ldots, x_{n}\right)$ or $\neg R\left(x_{1}, \ldots, x_{n}\right)$, the game ends.

- If $\varphi$ is $\psi_{1} \wedge \psi_{2}$, then $\forall$ belard chooses a conjunct $\psi_{i}$ and game $G^{S G S}\left(\psi_{i}, M\right)$ is played from position $s_{\psi_{i}}$.

- If $\varphi$ is $\psi_{1} \vee / W \psi_{2}$, then $\exists$ loise chooses a disjunct $\psi_{i}$ and game $G^{S G S}\left(\psi_{i}, M\right)$ is played from position $s_{\psi_{i}}$.

- If $\varphi$ is $\forall x \psi$, then $\forall$ belard chooses a value $a$ for $x$ from $M$, and game $G^{S G S}(\psi, M)$ is played from position $s(a / x) \psi$

- If $\varphi$ is $(\exists x / X) \psi$, then $\exists$ loise chooses a value $a$ for $x$ from $M$, and game $G^{S G S}(\psi, M)$ is played from position $s(a / x)_{\psi}$.

Definition 7 (Janssen (2001), 4.4; Janssen (2002), 10.5)

For each of $\exists$ loise's operators in $G^{S G S}(\varphi, M)$, a strategy is a function of one of the following forms:

- If $\varphi=\psi \vee_{/ W} \chi$ a function $F_{\varphi}: P_{\varphi} \rightarrow\{L, R\}$.

- If $\varphi=(\exists x / X) \chi$ a function $F_{\varphi}: P_{\varphi} \rightarrow M$.

( $L, R$ being two arbitrary distinct objects).

Now, given a set $W$ of variables occurring in an $I F$ formula $\varphi$, we can define $D_{W}$ as the set of variables that depend on variables from $W$ in formula $\varphi$. The definition is ambiguous, in that it might include only the variables whose strategy functions have variables from $W$ among its parameters; or, instead, it might take into account also indirect dependencies (e.g., if $w \in W$, $u$ depends on $w$, and $v$ depends on $u$, then $v \in D_{W}$ ). We will lean towards this second interpretation, although it must be said that the original text itself (Janssen (2001)) is ambiguous in this respect. Remembering that we write $t \sim_{W} s$ to mean that assignments $t$ and $s$ coincide everywhere except possibly on variables from $W$, winning positions and winning strategies are defined as follows:

Definition 8 (Janssen (2001), 4.5, although there the definition only applies to singleton slash sets; the clauses for $\wedge$ and $\forall$ are our addition)

We define winning strategies and winning positions for the game $G^{S G S}(\varphi, M)$ by simultaneous induction:

- If $\varphi=R\left(x_{1}, \ldots, x_{n}\right)$ then $s$ is a winning position if $\left(s\left(x_{1}\right), \ldots, s\left(x_{n}\right)\right) \in$ $R^{M}$

- If $\varphi=\neg R\left(x_{1}, \ldots, x_{n}\right)$ then $s$ is a winning position if $\left(s\left(x_{1}\right), \ldots, s\left(x_{n}\right)\right) \notin$ $R^{M}$.

- If $\varphi=\psi \vee_{/ W} \chi$, then $s$ is a winning position if there is a strategy $F_{\varphi}$ satisfying three conditions:

1. If $t \sim_{W \cup D_{W}} s$, then $F_{\varphi}(t)=F_{\varphi}(s)$.

2. If $F_{\varphi}(s)=L$, then $s_{\psi}$ is a winning position in game $G^{S G S}(\psi, M)$; if $F_{\varphi}(s)=R$, then $s_{\chi}$ is a winning position in game $G^{S G S}(\chi, M)$. 
3. If $t \sim_{W} s$, then there is an $r \sim_{D_{W}} t$ such that one of the following holds:

- $F_{\varphi}(t)=L$ and $r_{\psi}$ is a winning position for $G^{S G S}(\psi, M)$.

$-F_{\varphi}(t)=R$ and $r_{\chi}$ is a winning position for $G^{S G S}(\chi, M)$.

Such a strategy is a winning strategy for this game.

- If $\varphi=\psi \wedge \chi$, then $s$ is a winning position iff both $s_{\psi}$ and $s_{\chi}$ are winning positions for $G^{S G S}(\psi, M)$, resp. for $G^{S G S}(\chi, M)$.

- If $\varphi=(\exists y / W) \psi$ then $s$ is a winning position if there is a strategy $F_{\varphi}$ satisfying three conditions:

1. If $t \sim_{W \cup D_{W}} s$, then $F_{\varphi}(t)=F_{\varphi}(s)$.

2. $s\left(F_{\varphi}(s) / y\right)_{\psi}$ is a winning position in game $G^{S G S}(\psi, M)$.

3. If $t \sim_{W} s$, then there is an $r \sim_{D_{W}} t$ such that $r\left(F_{\varphi}(s) / y\right)_{\psi}$ is a winning position.

Such a strategy is a winning strategy for this game.

- If $\varphi=\forall y \psi$, then $s$ is a winning position if and only if, for all $a \in M$, $s(a / y)_{\psi}$ is a winning position.

Definition 9 (implicit in Janssen (2001))

An $I F$ sentence $\varphi$ is true iff the empty assignment $\emptyset$ is a winning position for $G^{S G S}(\varphi, M)$ according to Definition 8 . In such case we write $M \models_{S G S^{1}} \varphi$.

Dual definitions could be given of $\forall$ belard's winning positions and strategies and thus of falsity.

Notice that, apart from some extra conditions on which we shall dwell below, the core idea of Subgame Semantics is, as it was the case for Subjective Strategy Semantics, to require strategies to be uniformly winning over subforests generated by some information states. Thus, the use of the word "subgame" is somewhat improper and in contrast with game-theoretical literature (where a subgame is usually required to have only one root, and to be closed with respect to the indistinguishability relation).

A first general observation that we must make is that this semantics still falsifies Väänänen's example $\exists x(\exists y /\{x\})(x=0)$ (see again Figure 5). Indeed, the only winning choice for $x$ is $0^{M}$. Let us check point 3 of the existential clause. We have $\left(x, 0^{M}\right) \sim_{x}(x, b)$, and the $\sim_{\emptyset}$-class of $(x, b)$ is a singleton; but neither $(x, b),\left(y, 0^{M}\right)$ nor $(x, b),(y, b)$ is a winning position for the game of $x=0$.

So, also this semantics is counterintuitive. But this system has a peculiarity, the following result by Janssen:

Proposition 1 (Janssen (2001), 5.2) Let $\varphi$ be a Henkin sentence, that is a sentence of the form $\forall x \exists y \forall z(\exists w /\{x\}) \psi$. Then $M \models_{I F} \varphi$ iff $M \models_{S G S^{1}} \varphi$.

The importance of this result can be understood knowing that Henkin's partially ordered quantifier $H_{2}^{1}=\left(\begin{array}{l}\forall x \exists y \\ \forall z \exists w\end{array}\right)$ (Henkin (1961)), added to firstorder logic with empty signature, can express higher-order concepts, such as infinity. As a side note, the correct expression of a branching quantification 
should be $\forall x \exists y \forall z(\exists w /\{x, y\})$ (notice the extra $y$ in the slash set); but, in our opinion, Janssen's proof is not spoiled by this mistake. So, we have as an immediate consequence that

Proposition 2 IF logic with $S G S^{1}$ semantics is not equiexpressive to a fragment of first-order logic.

The fact that the meaning of a formula is expressed in terms of sets of assignments, and not sets of sets of assignments, suggests however that this semantics, most likely, does not have full $\Sigma_{1}^{1}$ expressive power (cp. with the results of Cameron and Hodges (2001)).

Let us compare briefly this system with first-order logic and with Subjective Strategy semantics. Notice that the $S G S^{1}$ clauses for atomic formulas, negations, conjunctions and universal quantification, are taken care of by the classical notion of winning strategy, plus the restriction of locality (strategies depend only on variables occurring in the subformula under consideration); for first-order formulas, this restriction is irrelevant. Similarly, in the disjunctive clauses, the locality restriction and clauses 2. and 3. are irrelevant for first-order formulas; thus, we can conclude that $F O \subset I F_{S G S^{1}}$.

The comparison with Subjective Strategy semantics is more problematic. $I F_{S S S}$ might perhaps be immune from signalling, but we have no reason to think that it enjoys locality; the restriction of locality is instead imposed in $I F_{S G S^{1}}$, making games more difficult to win in $I F_{S G S^{1}}$ than in $I F_{S S S}$. On the contrary, the clauses 3 . (for disjunctions and existential quantifiers) of $I F_{S G S^{1}}$ impose more liberal restrictions on winning strategies than subjectivity of the winning strategies (the latter condition requires a strategy to be winning in all indistinguishable nodes; the former only requires that for some such nodes). So, we are inclined to think that the two logics are incomparable.

We make some more specific comments about the semantic clauses for $\mathrm{V} / W$ and $(\exists y / Y)$.

It is noteworthy that condition 1. (of both clauses) states that the choice function should be independent not only from the variables occurring in the slash sets, but also from variables which depend on the variables from the slash set (this is obtained by adding $D_{W}$ to the parameters of the indistinguishability relation). We may call this the Transitive Closure condition. This is a marked difference with standard $I F$ semantics, and it yields as a consequence that many possible forms of signalling are inhibited.

Reading Janssen's commentary to his own semantics (see in particular Janssen (2001), 3.4-3.5), we got the impression that prescription 3. is meant to embody the following Covariance Condition (which, for simplicity, we only express for singleton slash sets); this is fundamentally a way to prohibit signalling. Notice also that the objects $c_{x}, c_{y}, \mathbf{b}$ mentioned below are not necessarily arguments of the strategy functions which are named, but might be arguments of some subterm.

CC. If a subformula is of the form $(\exists y /\{x\}) \psi$, and

$$
\left\{\left(x, c_{x}\right),\left(v_{1}, f_{v_{1}}\left(\ldots, c_{x}, \ldots\right)\right), \ldots,\left(v_{n}, f_{v_{n}}\left(\ldots, c_{x}, \ldots\right)\right),\left(y, c_{y}\right), \ldots\right\}
$$


where $v_{1}, \ldots, v_{n}$ are all the variables which directly or indirectly depend on $x$, is a winning position for $\psi$, then also

$$
\left\{(x, \mathbf{b}),\left(v_{1}, f_{v_{1}}(\ldots, \mathbf{b}, \ldots)\right), \ldots,\left(v_{n}, f_{v_{n}}(\ldots, \mathbf{b}, \ldots)\right),\left(y, c_{y}\right), \ldots\right\}
$$

is a winning position, for any $\mathbf{b} \in M$.

This condition stipulates that, if the choice for $y$ is winning in the current play of the game, then it should have also been winning if a different value had been chosen for $x$ (and, consequently, the strategies adopted had modified accordingly the values of all variables that depend on $x$ ).

It is not evident that CC may be really enforced by condition 3 ; we suspect it does, but lack a proof.

We think this Covariance Condition to be a rather reasonable and somewhat neglected requirement to impose on a notion of independence; it could perhaps be interesting to introduce a variant of Subgame Semantics which explicitly includes this clause. The Transitive Closure Condition, instead, blocks any form of signalling and induces a literal observance of the independence requirements.

\section{Subgame Semantics, second version}

The second version of Subgame Semantics differs from the prototype in the following clauses, coming from Janssen (2002), 10.4, and in some lesser detail which we shall ignore here:

- If $\varphi=\psi \vee / W \chi$ then $s$ is a winning position for $G(\varphi, M)$ if there is a strategy $F_{\varphi}$ satisfying three conditions:

1. If $t \sim_{W} s$, then $F_{\varphi}(t)=F_{\varphi}(s)$.

2. If $F_{\varphi}(s)=L$, then $s_{\psi}$ is a winning position in game $G^{S G S}(\psi, M)$; if $F_{\varphi}(s)=R$, then $s_{\chi}$ is a winning position in game $G^{S G S}(\chi, M)$.

3. If $t \sim_{W} s$, then one of the following holds:

- Neither $t_{\psi}$ is a winning position for $G^{S G S}(\psi, M)$, nor $t_{\chi}$ is a winning position for $G^{S G S}(\chi, M)$.

- $F_{\varphi}(t)=L$ and $t_{\psi}$ is a winning position for $G^{S G S}(\psi, M)$.

$-F_{\varphi}(t)=R$ and $t_{\chi}$ is a winning position for $G^{S G S}(\chi, M)$.

Such a strategy is a winning strategy for this game.

- If $\varphi=(\exists y / W) \psi$ then $s$ is a winning position if there is a strategy $F_{\varphi}$ satisfying three conditions:

1. If $t \sim_{W} s$, then $F_{\varphi}(t)=F_{\varphi}(s)$.

2. $s\left(F_{\varphi}(s) / y\right)_{\psi}$ is a winning position in game $G^{S G S}(\psi, M)$.

3. If $t \sim_{W} s$, then, if there is a $c \in M$ such that $t(c / y)_{\psi}$ is a winning position, then $t\left(F_{\varphi}(s) / y\right)_{\psi}$ is a winning position for $G^{S G S}(\psi, M)$.

Such a strategy is a winning strategy for this game.

If in a certain position $s$ there is no move which leads to a winning position, we may say that $s$ is a losing position. So, we may restate clause 3 . for existential formulas as stating that the requested function must be a winning 
strategy in all positions which are indistinguishable from the current one and which are not losing positions. This last specification (called "clause 3b." by Janssen) was devised to treat properly sentences like Väänänen's example $\exists x(\exists y /\{x\})(x=0)$, which would otherwise have a totally counterintuitive meaning.

We shall call this semantics $S G S^{2}$. Besides the introduction of clause $3 \mathrm{~b}$, the remarkable difference with $S G S^{1}$ is the absence of any requirement in the direction of the Transitive Closure Condition or the Covariance Condition. We wonder why the author may have abandoned these important intuitions; perhaps he deemed them to be overly complicated and intractable clauses. Or, he might have seen a problem in the fact that a precise definition of these clauses involves accounting for variables (those in $D_{W}$ ) that are quantified not in the subformula associated to the subgame under consideration, but rather in the sentence that contains it; this ruins the dream of a compositional semantics.

In any case, the Covariance Condition should be reformulated to take into account clause $3 \mathrm{~b}$ :

CC'. If a subformula is of the form $(\exists y /\{x\}) \psi$, and

$$
\left\{\left(x, c_{x}\right),\left(v_{1}, f_{v_{1}}\left(\ldots, c_{x}, \ldots\right)\right), \ldots,\left(v_{n}, f_{v_{n}}\left(\ldots, c_{x}, \ldots\right)\right),\left(y, c_{y}\right), \ldots\right\}
$$

where $v_{1}, \ldots, v_{n}$ are all the variables which directly or indirectly depend on $x$, is a winning position for $\psi$, then also

$$
\left\{(x, \mathbf{b}),\left(v_{1}, f_{v_{1}}(\ldots, \mathbf{b}, \ldots)\right), \ldots,\left(v_{n}, f_{v_{n}}(\ldots, \mathbf{b}, \ldots)\right),\left(y, c_{y}\right), \ldots\right\}
$$

is a winning position, for any $\boldsymbol{b}$ such that there is a winning position

$$
\left\{(x, \mathbf{b}),\left(v_{1}, f_{v_{1}}(\ldots, \mathbf{b}, \ldots)\right), \ldots,\left(v_{n}, f_{v_{n}}(\ldots, \mathbf{b}, \ldots)\right),(y, \mathbf{e}), \ldots\right\} .
$$

Or, perhaps, it would be reasonable to add to these kinds of semantics a different covariance requirement, which takes into account, for clause $3 \mathrm{~b}$, also the correlation of variables which depend on the variable $y$ which is chosen in the current stage; this is obtained by replacing the last proviso above with (in slightly simplified notation):

$$
\left\{(x, \mathbf{b}),(y, \mathbf{e}),\left(v_{i}, f_{v_{i}}(\ldots, \mathbf{b}, \mathbf{e}, \ldots)\right), \ldots\right\} .
$$

We can explicitly show with an example that CC' is violated in $S G S^{2}$.

Example 4 Consider the sentence $\varphi:=\forall x \exists y(x=y \wedge(\exists u /\{x\})(u>y))$ over the structure of natural numbers.

We argue that this sentence, on an intuitive reading and in observance of the Covariance Conditions, should be non-true in $\mathbb{N}$.

Indeed, suppose $\forall$ belard chooses an element $a$ for $x$; ᄏloise must respond with the identity function $f_{y}(x):=x$ and with some function $f_{u}(y)$ whose value is greater than its argument (e.g. $\left.f_{u}(y):=y+3\right)$. By the Covariance Condition, if we keep fixed the value $b:=f_{u}(a)$ and change, for example, the 
value assigned to $x$ into $a^{\prime}:=a+5$ then the value assigned to $y$ becomes $f_{y}\left(a^{\prime}\right)$, that is, $a^{\prime}$, and there we have no more $u>y$. Thus, ᄏloise has no winning strategies for $\varphi$.

In contrast with this, we prove the truth of the sentence $\varphi$ according to $S G S^{2}$ semantics by demonstrating that the empty assignment is a winning position for $\varphi$ on $\mathbb{N}$. Here we have that:

- By ordinary semantics, the set of winning positions corresponding to the formula $x=y$ is

$$
W \operatorname{Pos}\left(" x=y^{\prime \prime}\right)=\{\{(x, a),(y, b)\} \mid a, b \in \mathbb{N}, a=b\}
$$

and also

- WPos(" $\left(^{\prime \prime}>y^{\prime \prime}\right)=\{\{(y, b),(u, c)\} \mid b, c \in \mathbb{N}, c>b\}$.

- WPos(" $\left.(\exists u /\{x\})(u>y)^{\prime \prime}\right) \subseteq\{\{(x, a),(y, b)\} \mid a, b \in \mathbb{N}\}$.

We prove that the inclusion is an equality. Let us check the semantic conditions of the existential clause.

2) By the strategy $f_{u}(\{(y, b)\}):=b+1$ we can extend the assignment $\{(x, a),(y, b)\}$ to an assignment $\{(x, a),(y, b),(u, b+1)\} \in W \operatorname{Pos}\left({ }^{\prime \prime} u>y^{\prime \prime}\right)$.

1) $f_{u}$ does not depend on the slashed variable $x$.

3 ) If we assign to $x$ a new value $a^{\prime}$, then $\{(x, a),(y, b),(u, b+1)\}$ is still a winning position. (Notice that the value assigned to $y$ has not changed according to the new value of $x$ )

Thus,

$$
W \operatorname{Pos}\left({ }^{\prime \prime}(\exists u /\{x\})(u>y)^{\prime \prime}\right)=\{\{(x, a),(y, b)\} \mid b \in \mathbb{N}\} .
$$

- WPos(" $\left(^{\prime \prime}=y \wedge(\exists u /\{x\})(u>y)^{\prime \prime}\right)=\{\{(x, a),(y, b)\} \mid a=b\}$, since these assignments must satisfy the defining condition of (2), and also the trivial defining condition of (3).

- WPos(" $\left.\exists y(x=y \wedge(\exists u /\{x\})(u>y))^{\prime \prime}\right)=\{\{(x, a)\} \mid a \in \mathbb{N}\}$ since the strategy function $f_{y}(\{(x, a)\}):=a$ allows extending the assignment $\{(x, a)\}$ to a couple $\{(x, a),(y, a)\} \in W \operatorname{Pos}\left(" x=y \wedge(\exists u /\{x\})(u>y)^{\prime \prime}\right)$.

- Thus, $W \operatorname{Pos}\left({ }^{\prime \prime} \forall x \exists y(x=y \wedge(\exists u /\{x\})(u>y))^{\prime \prime}\right)=\{\emptyset\}$. The sentence is true in $\mathbb{N}$.

This argument shows that $S G S^{2}$ semantics, although it takes into account independence from slashed variables (in this case, $x$ ), does not account for independence from variables which should be adjusted together with $x$ (in this case, $y$; failing to adjust it together with $x$ may falsify the conjunct $x=y$ ). It would be interesting, perhaps, to devise a new version of Subgame Semantics that has both clauses 3. from $S G S^{2}$ and 1. from $S G S^{1}$, and which is thus likely both to have the $\mathrm{CC}$ property and not to give very counterintuitive values to sentences; we will not pursue here this possibility.

Finally, it must be remarked that the sentence considered in this example is not true in $S G S^{1}$ semantics; so, it does not disprove our claim that $S G S^{1}$ respects CC. 
Let us close this parenthesis, and go back analyzing $S G S^{2}$. It is clear that first-order formulas keep their standard values under this semantics. As in the case of Subjective Strategy Semantics, and in contrast with what we said about $S G S^{1}$, we can state that:

Theorem 1 IF logic with $S G S^{2}$ semantics is equiexpressive with first-order logic.

Proof (Sketch)

This is fundamentally an argument of Hodges (2012). Each $I F$ sentence $\varphi$ evaluated under $S G S^{2}$ semantics can be translated into a first-order sentence $\varphi$ by replacing each existential subformula $(\exists y / W) \psi$ with

$$
\exists y(\psi \wedge \forall \bar{w}(\exists s \psi[s / y] \rightarrow \psi))
$$

(where $\bar{w}$ is $W$ written as a sequence); and replacing each disjunctive subformula $\psi \vee / W \chi$ with

$$
(\psi \wedge \forall \bar{w}((\neg \psi \wedge \neg \chi) \vee \psi)) \vee(\chi \wedge \forall \bar{w}((\neg \psi \wedge \neg \chi) \vee \chi))
$$

\section{Rationalization}

As far as we know, after Janssen (2002) there have not been further developments of SGS semantics in the literature. However, there have been some attempts to find alternative semantics or game-theoretical justifications of Subgame Semantics. All these attempts were based on the game-theoretical notion of rationalizability (weak domination). The common idea in these kinds of semantics is that of considering the semantic games not as (extensive) 2-player games, but rather as (strategic) multiplayer games. A strategic game can be formalized as a triple $\left(N,\left\{S_{i}\right\}_{i \in N},\left\{u_{i}\right\}_{i \in N}\right)$, where $N$ is the set of players, $S_{i}$ a set of strategies for player $i$, and $u_{i}: \prod_{i \in N} S_{i} \rightarrow \mathbb{R}$ the payoff function of player $i$. In our specific case, the player $i$ corresponding to a certain (occurrence of) logical operator has as its strategy set $S_{i}$ the set of allowed strategy functions (for the extensive game) corresponding to that (occurence of) logical operator; the players associated to existential quantifiers and disjunction have, each, the same payoff (to be defined precisely below) as Đloise has in the 2-player games ( 1 if she wins, 0 if she looses); and universal and conjunction players have the payoff of $\forall$ belard. Yet, as stated explicitly in Sevenster (2007), these semantics are not thought out in order to make the players behave as if they formed two teams, each of which is coordinated by a common strategy; rather, the players should act as rational individuals who choose the most convenient individual strategies, but have not the possibility to coordinate themselves with other players. 
The first two examples ${ }^{9}$ of these kinds of semantics appeared in Sevenster (2007). We mention them only briefly, since it is clear that they are inadequate semantics and only embryonal attempts towards a semantics that is described in more clear terms in publications of Janssen (Janssen (2005), Janssen (2007)).

The first of these attempts was based on the notion of weak dominance. We consider the semantic games of $I F$ logic as games between two teams of players (one player for each logical operator, except for negations). Each single player wins the game if his team wins. Given a game $G(\varphi, M)$, we call profile a sequence $\pi$ of strategy functions, one for each of the players in the game. Of course, we can always identify a profile with a pair $(\sigma, \tau)$, where $\sigma$ is a strategy for the $\exists$ loise team and $\tau$ is a strategy for the $\forall$ belard team. We obtain a strategic game from this extensive game by defining the payoff function of player $i$ on profiles of $G(\varphi, M)$ :

$$
u_{i}(\pi)=\left\{\begin{array}{l}
1 \text { if } i \text { wins when players follow } \pi \\
0 \text { otherwise }
\end{array}\right.
$$

The strategies of this strategic game are the strategy functions of the corresponding extensive game.

We also write $u_{\exists}$ for $u_{i}$ if $i$ is an $\exists$ loise, and $u_{\forall}$ if $i$ is an $\forall$ belard player. Furthermore, we follow the conventions of game theory in writing $\pi_{i}$ for the strategy (function) for player $i$ in profile $\pi$; in writing $\pi_{-i}$ for the (partial) profile obtained removing from $\pi$ the strategy (function) $\pi_{i}$ of player $i$; and in writing $\left(\pi_{-i}, f\right)$ for the profile obtained replacing, in $\pi$, the strategy (function) $\pi_{i}$ with $f$.

Definition 10 A strategy $f_{i}$ is weakly dominant if, for any strategy profile $\pi$

$$
u_{\exists}\left(\pi_{-i}, f_{i}\right) \geq u_{\exists}(\pi) .
$$

This notion of dominance is used to define a semantics in the following way: we say that sentence $\varphi$ is true in $M\left(M=_{W D} \varphi\right)$ if there is a combination $\sigma$ of strategies for the $\exists$ loise players such that each component of $\sigma$ is weakly dominant for its player, and such that, for all combinations of strategies $\tau$ of the $\forall$ belard players, $u_{\exists}(\sigma, \tau)=1$. One weak point of this definition lies in the notion of dominance that was chosen; not many games have weakly dominant strategies. As a result, this semantics is strictly weaker than first-order logic and even falsifies some classical validities.

The second idea in Sevenster's paper is based on iterated removal of weakly dominated strategies (Sevenster (2007), sec. 5); if all possible combinations of the surviving strategies are winning for $\exists$ loise players, then the sentence is true in the model under consideration $\left(M \models_{I W D} \varphi\right)$. This idea seems far more

\footnotetext{
9 Actually, the paper Janssen (2005) of Janssen appeared earlier, but from acknowledgements occurring in his paper, the precedence of Sevenster's attempts is evident. See also the Bibliographical Note at the end of the paper for even earlier suggestions coming from the works of van Benthem.
} 
reasonable than considering the existence of such strategy profiles (and we will reconsider it in the next section); yet, somewhat bizzarrely, the definition of the removal process seems to depend on the sentence being in prenex normal form, and on the extensional, rather than strategic structure of games: the specific process of iterated removal is indeed determined by the order in which variables are quantified in the sentence. In the next section, we will consider an order-independent variant of this notion.

The next contribution we shall examine comes again from Janssen. The system we are going to describe figures in two distinct but similar papers, "Independence Friendly Logic as a strategic game" (Janssen (2005)) and "Independence and Hintikka games" (Janssen (2007)). This semantics is based on rationalization, applied to the multiagent game.

Definition 11 A strategy $g_{i}$ for player $i$ is weakly dominated by $f_{i}$ if for every profile $\pi$

$$
u_{i}\left(\pi_{-i}, f_{i}\right) \geq u_{i}\left(\pi_{-i}, g_{i}\right)
$$

and for some profile $\hat{\pi}$

$$
u_{i}\left(\hat{\pi}_{-i}, f_{i}\right)>u_{i}\left(\hat{\pi}_{-i}, g_{i}\right)
$$

A strategy for player $i$ is rational if it is not weakly dominated by any other strategy of the same player.

Definition 12 We state that a sentence $\varphi$ is true in a structure $M$, and we write $M \models_{R} \varphi$, if for any combination $\sigma$ of rational strategies of the $\exists$ loise players, and any combination $\tau$ of rational strategies of the $\forall$ belard players, $u_{\exists}(\sigma, \tau)=1$.

For any finite structure $M$, we have

$$
M \models{ }_{R} \varphi \Rightarrow M \models \varphi
$$

where the second assertion expresses standard IF semantics. Indeed, the relation " $g$ is weakly dominated by $f$ " is a strict partial order on the set of strategies of a player; if the structure is finite, so is the set of strategies of each player; consequently, the set $P$ of profiles constituted of rational strategies is non-empty. Then, if $M=_{R} \varphi$ holds, any collective strategy for the $\exists$ loise players which is extracted from a profile in $P$ is a winning strategy.

An interesting aspect of this semantics, from the point of view of linguistics, is that it assigns more reasonable interpretations to some variant of the classical townsman/villager example of branching quantification (see Janssen (2007), sect.6).

Comparing this semantics with $W D$, we have here a (more reasonable) universal requirement instead of an existential one. But notice that, as observed in Janssen (2007), this kind of semantics becomes very paradoxical when applied to infinite structures; for example, sentence

$$
\theta:=\exists x \exists y(y<x) \wedge \exists z \forall w(z=w)
$$


is unreasonably true in $\mathbb{N}$ (each strategy for $x$ is dominated by strategies which choose a greater value; so, no profiles survive).

Two positive results are stated in Janssen's paper. The first one asserts that any rational strategy can be replaced by an equivalent strategy which depends only on the variables occurring in the subformula under consideration. This should point to the existence of a formulation of the semantics which is both compositional and local, in the sense that the meaning of a subformula does not depend on the context in which it occurs. This result is rather fishy, since no definition is given of rationality for the strategy associated to an open formula. Furthermore, the proof of the statement looks rather suspect, since it seems not to use the hypothesis that certain variables do not occur in the subformula.

The second result, depending on the first, is precisely a compositional clause for existential formulas. We need to specify what it means for an assignment $s$ to satisfy a formula: writing $M=\varphi(\bar{x})[s]$ we mean that all rational strategies are winning, when played against any collection of moves, for the sentence which is obtained replacing the free variables $\bar{x}$ with $s(\bar{x})$. (It is clear that here Janssen has in mind a first-order compositional semantics).

The compositional clause for existential sentences is as follows (with a small correction on our part $\left.{ }^{10}\right)$ :

$$
\begin{gathered}
M \models(\exists y /\{\bar{x}\}) \psi(\bar{x}, y)[s] \\
\mathbb{\Downarrow} \\
\text { there is a function } f: F V(\psi) \rightarrow M \text { such that } \\
M \models(\psi(\bar{x}, f(\bar{x})) \wedge \forall \bar{z}(\exists u \psi(\bar{z}, u) \rightarrow \psi(\bar{z}, f(\bar{z}))))[s],
\end{gathered}
$$

$\bar{z}$ being a sequence of new variables. As Janssen observed, this clause has a very strong resemblance to the corresponding clause of $S G S^{2}$; this led him to suggest that the two semantics may coincide, and that $R$ semantics may be a game-theoretical justification of $S G S^{2}$. In the following, we shall disprove this claim: sentence $\zeta$ from example 13 shows that $R$ and $S G S^{2}$ do not coincide, even on finite structures; the sentence (4) mentioned above provides a counterexample in infinite structures, and it also excludes the possibility that the set of truths of $I F_{R}$ be a subset of the truths of $I F_{S G S^{2}}$. This seems also to suggest that the compositional clause just exposed may be uncorrect. (In any case, it is not general enough, since it seems to cover only formulas $\psi$ which contain at most one free variable besides those listed in the slash set).

\section{Dominance solvability}

It may be natural, at this point, to restrict further the set of strategies considered by Janssen by iterating the removal of weakly dominated strategies.

\footnotetext{
10 In the original text, $z$ is written as a single variable, not a vector; and in the left term of the implication, $\bar{x}$ took the place of our $\bar{z}$.
} 
This can be done in a more reasonable way than the procedure described in Sevenster (2007); strangely enough, this line of thought has not been pursued in the literature, and we introduce it here. The idea emerged during some conversations between Pietro Galliani and the author; we were not aware, at the time, of the affine publications of Sevenster and Janssen.

Definition 13 Let $G(\varphi, M)$ be a semantic game, with $N=\{1, \ldots, n\}$ its set of players, defined as before, and let $S_{i}^{0}$ be the set of strategies of player $i$. We inductively define

$$
\begin{gathered}
G_{0}(\varphi, M):=G(\varphi, M) \\
G_{k+1}(\varphi, M):=\left\{N,\left\{S_{1}^{k+1}, \ldots, S_{n}^{k+1}\right\},\left\{u_{i}\right\}_{i \in N}\right\}
\end{gathered}
$$

where $S_{i}^{k+1}$ is the subset of $S_{i}^{k}$ which is constituted by the strategies which are rational for player $i$ in $G_{k}(\varphi, M)$.

Let $G_{D S}(\varphi, M)$ be the fixed point of this procedure ${ }^{11}$.

Definition 14 We say that a sentence $\varphi$ is true in a structure $M$, and we write $M \models{ }_{D S} \varphi$ if for all profiles $\sigma$ in $G_{D S}(\varphi, M), u_{\exists}(\sigma)=1 .^{12}$

This notion of dominance is based on simultaneous removal of all strategies, of all players, which are weakly dominated; and on an iteration of the process. It is required for truth that all the profiles that can be formed from the surviving strategies be winning for $\exists$ loise players. Falsity (and thus undeterminedness) may be defined analogously.

This solution concept shares the same dubious interpretational status of all procedures of removal of weakly dominated strategies. Yet it seems to us to be the most natural among such kind of processes; and at least it is not dependent on the extensive structure of the game, as the procedure related to $I W D$ semantics is. Furthermore, the present procedure seems to have a special place in the literature, and even a name: dominance solvability (Moulin (1979), and see also Osborne and Rubinstein (1994), Exercise 63.2). Some recent results from epistemic game theory (Halpern and Pass (2009)) seem to suggest that dominance solvability is the correct solution concept for the description of a game in which each player only knows, about other players, that they are rational. Our intendended epistemic interpretation differs from such a setup in that players know something more beyond the rationality of all players: they know the type of each player, that is, his/her payoff function. This suggest that $D S$ might not yet capture the epistemic intuition; so, in the next section we will consider a clause that seems to be more appropriate.

Example 5 Consider sentence $\varphi:=\exists x \exists y(x=y)$, and a structure $M=\{a, b\}$. We list in the following table all the strategies available to the two players. We denote as $i d: M \rightarrow M$ the identity function, and as switch $: M \rightarrow M$ the switching strategy $\operatorname{switch}(a):=b, \operatorname{switch}(b):=a$.

11 Also known, in the literature, as the set of rationalizable strategies, due to a characterization theorem of Pearce (1984).

12 As in the previous section, $u_{\exists}$ denotes the payoff function of any $\exists$ loise player. 


\begin{tabular}{|l|l|}
\hline$\exists x$ & $\exists y$ \\
\hline$s_{1}: a$ & $t_{1}: a$ \\
$s_{2}: b$ & $t_{2}: b$ \\
& $t_{3}: i d(x)$ \\
& $t_{4}: \operatorname{switch}(x)$ \\
\hline
\end{tabular}

Strategies $t_{1}, t_{2}$ and $t_{4}$ of player $\exists y$ are all weakly dominated by strategy $t_{3}$. So, the game reduces to $G_{1}(\varphi, M)$, characterized by the following strategies.

\begin{tabular}{|l|l|}
\hline$\exists x$ & $\exists y$ \\
\hline$s_{1}: a$ & $t_{3}: i d(x)$ \\
$s_{2}: b$ & \\
\hline
\end{tabular}

No strategies in this game are weakly dominated anymore, so $G_{D S}(\varphi, M)=$ $G_{1}(\varphi, M)$. For this game two strategy profiles are available: $\left(s_{1}, t_{3}\right)$ and $\left(s_{2}, t_{3}\right)$. Both are wins for $\exists$ loise players (i.e., $\left.u_{\exists}\left(s_{2}, t_{3}\right)=u_{\exists}\left(s_{1}, t_{3}\right)=1\right)$, so $M \models_{D S}$ $\exists x \exists y(x=y)$.

Example 6 The following sentences

$$
\begin{gathered}
\exists x(\exists y /\{x\})(x=y) \\
\forall x \exists z(\exists y /\{x\})(x=y) \\
\forall x \exists z(\exists y /\{x, z\})(x=y)
\end{gathered}
$$

are all non-true for a common reason: no strategy for any player is weakly dominated, but the profiles in the games are not all wins for $\exists$ loise.

Example 7 However, for infinite structures we have similar problems as for $I F_{R}$. The sentence (4) of the previous section is again true for trivial reasons. This proves that dominance solvability is not a game-theoretical justification of Subgame Semantics. Yet, we cannot exclude the possibility that it is the correct justification over finite structures.

We obviously have

\section{Theorem 2}

$$
M \models{ }_{R} \varphi \Rightarrow M \models{ }_{D S} \varphi
$$

In section 11, we will show that the inclusion $I F_{R} \subseteq I F_{D S}$ is strict (Example 12). 


\section{Cartesian semantics}

We introduce here a new semantics which is related to some solution concepts which were discussed, for example, in Luce and Raiffa (1957). It is founded on an intuition of what it means for a set of rational players to have a winning strategy when they share the same utility function but they cannot communicate among themselves. This semantics will be defined not only for $I F$ languages, but also for the more general $C S$ languages described above, and which we define more formally here.

An $I F$ sentence is said to be strongly regular if no variable is quantified more than once in it.

Definition 15 Let $\varphi$ be a strongly regular IF sentence. Replace each quantifier $(\exists v / V)$ occurring in $\varphi$ with $\left(\exists v / V_{-} U\right)$, where $U$ is a set of variables which are quantified before $v$. Then the resulting string is a $\boldsymbol{C S}$ sentence.

We reason in a similar framework as in the previous sections, associating a player to each quantifier and binary connective, and partitioning them into $\exists$ loise and $\forall$ belard players, with the usual utility functions.

We want to model a semantic concept that expresses the fact that the existential players have a method for winning (getting payoff 1) under the following epistemic conditions:

1) Each player knows that her comrades share the same payoff function, and that they play rationally.

2) Each player only knows the strategy functions applied by those players whose associated variable does not occur in her underscore set, and in whose underscore set she does not appear.

Notice that in 2) we are postulating that players are always unaware of the strategies employed by players whose quantifier or connective occurs subordinated. Thus, declarations of (strategy-)independence will always state a condition of symmetrical independence. (Asymmetrical independencies surely have some theoretical interest, and should be investigated in future work).

We will try to express the idea of "having a winning method under conditions 1) and 2)" by requesting that the set of winning strategies has a peculiar form. For sure, it must be non-empty, but that is not enough! We want each player to be able to pick up one of his (potentially winning ${ }^{13}$ ) strategy functions trusting that, if all of his comrades do the same (which they must, by rationality), then the strategy obtained collecting all the strategy functions of the members of the team is a winning strategy. We think that this can be achieved only if the set of winning strategies satisfies very restrictive conditions, which we are going to describe. Essentially, the idea is that,

\footnotetext{
13 We say that a strategy function $f_{v}$ is potentially winning if it is part of a winning strategy, that is, there is a strategy $\sigma=\left(\ldots, f_{v}, \ldots\right)$ of $\exists$ loise such that, for every strategy $\tau$ of $\forall$ belard, $u_{\exists}(\sigma, \tau)=1$.
} 
$A$ ) whenever $u$ is in the underscore set of $v$ (or viceversa), the set of "best" uniform strategies of $\exists$ loise, restricted to the players $\exists u$ and $\exists v$, must be a cartesian product $B^{u} \times B^{v}$, so that it does not make a difference for each single $\exists$ loise player to choose among his set of "best" choices.

$B)$ The set of "best" strategies of the $\exists$ loise team is nonempty.

But what does it mean for a strategy to be a "best" one? For simplicity, we begin considering the set of all winning strategies of $\exists$ loise, until a counterexample will show us that this is not the right notion.

Let $\varphi$ be a $C S$ sentence, and assume $\left(\exists v_{1} / V_{i}\right), \ldots,\left(\exists v_{m} / V_{m}\right)$ is the list of existential quantifiers occurring in $\varphi$, from left to right; suppose each variable is quantified only once in $\varphi$. Let $\exists(\varphi)$ be the set of existentially quantified variables of $\varphi$. Let $\prec$ be the relation of superordination $\left(v_{i} \prec v_{j}\right.$ iff $v_{j}$ occurs in $\varphi$ in the scope of a quantifier over $v_{i}$ ). We define the independence relation

$$
\smile_{\varphi}:=\left\{(i, j) \mid v_{i} \prec v_{j} \wedge v_{i} \in V_{j} \wedge v_{i}, v_{j} \in \exists(\varphi)\right\}
$$

on indices of variables. Let $l=\left|\smile_{\varphi}\right|$. In order to express a condition on strategies, we make use of Skolem functions; we review here the context-dependent, first-order Skolemization of $I F$ sentences and formulas. We follow the definition 4.3 of Mann et al. (2011). Let $\psi$ be an $I F$ formula, $U \supseteq F V(\psi)$ a finite set of variables. Then:

$$
\begin{aligned}
& \operatorname{Sk}_{U}(\psi)=\psi(\psi \text { is a literal }) \\
& \operatorname{Sk}_{U}\left(\psi \vee \psi^{\prime}\right)=\operatorname{Sk}_{U}(\psi) \vee \operatorname{Sk}_{U}\left(\psi^{\prime}\right) \\
& \operatorname{Sk}_{U}\left(\psi \wedge \psi^{\prime}\right)=\operatorname{Sk}_{U}(\psi) \wedge \operatorname{Sk}_{U}\left(\psi^{\prime}\right) \\
& \operatorname{Sk}_{U}\left(\left(\exists v_{i} / V_{i}\right) \psi\right)=\operatorname{Sub}\left(\operatorname{Sk}_{U \cup\left\{v_{i}\right\}}(\psi), v_{i}, h_{i}\left(y_{1}, \ldots, y_{n}\right)\right) \\
& \operatorname{Sk}_{U}\left(\left(\forall v_{i} / V_{i}\right) \psi\right)=\forall v_{i} \operatorname{Sk}_{U \cup\left\{v_{i}\right\}}(\psi) \\
& \operatorname{Sk}(\varphi):=\operatorname{Sk}_{\varnothing}(\varphi) \text { for } \varphi \operatorname{IF} \text { sentence. }
\end{aligned}
$$

where $y_{1}, \ldots, y_{n}$ enumerate the variables in $U \backslash V_{i} ; h_{i}$ is a new function symbol (Skolem function) associated to $v_{i}$; and the operation $S u b(\psi, x, t)$ is substitution of free occurrences of variable $x$ in $\psi$ with term $t$. In case $\varphi$ is a $C S$ sentence, denote as $\varphi^{\#}$ the corresponding $I F$ sentence which is obtained removing the underscore sets, and define $\operatorname{Sk}(\varphi):=\operatorname{Sk}\left(\varphi^{\#}\right)$.

If the function symbols $f_{1}, \ldots, f_{m}$ are thought as strategy functions for the variables $v_{1}, \ldots, v_{m}$ respectively, then we can express the fact that $\left(f_{1}, \ldots, f_{m}\right)$ is a collective winning strategy (uniform according to the slash sets) for the $\exists$ loise team by the following sentence $W \operatorname{Strat}_{\varphi}\left(f_{1}, \ldots, f_{m}\right)$ :

$$
\operatorname{Simsub}\left(\operatorname{Sk} \varphi,\left(h_{1}, \ldots, h_{m}\right),\left(f_{1}, \ldots, f_{m}\right)\right)
$$

that is, the simultaneous substitution, in $\operatorname{Sk} \varphi$, of each $f_{i}$ for the corresponding $h_{i}$. Let $\Omega_{\varphi}$ be the condition

$$
\left[\exists f_{1} \ldots \exists f_{m} \operatorname{WStrat}_{\varphi}\left(f_{1}, \ldots, f_{m}\right)\right] \wedge\left[\bigwedge_{(i, j) \in \smile_{\varphi}} \forall g_{1} \ldots \forall g_{m} \forall g_{i}^{\prime} \forall g_{j}^{\prime}(\psi \rightarrow \chi)\right]
$$


where

$$
\psi=W \operatorname{Strat}_{\varphi}\left(g_{1}, \ldots, g_{m}\right) \wedge W \operatorname{Strat}_{\varphi}\left(g_{1}, \ldots, g_{i}^{\prime}, \ldots, g_{j}^{\prime}, \ldots, g_{m}\right)
$$

and

$\chi=W \operatorname{Strat}_{\varphi}\left(g_{1}, \ldots, g_{i}, \ldots, g_{j}^{\prime}, \ldots, g_{m}\right) \wedge W \operatorname{Strat}_{\varphi}\left(g_{1}, \ldots, g_{i}^{\prime}, \ldots, g_{j}, \ldots, g_{m}\right)$.

Under the identification of "best" with "winning", the left conjunct expresses condition $B$, that the set $W$ of winning strategies is nonempty. If we call $S_{i}$ the set of strategies of player $i$, then for any set $\left\{i_{1}, \ldots, i_{n}\right\}$ we can talk of the $\left\{i_{1}, \ldots, i_{n}\right\}$-projection of $W, p_{\left\{i_{1}, \ldots, i_{n}\right\}}(W)=\left\{\left(f_{i_{1}}, \ldots, f_{i_{n}}\right) \in S_{i_{1}} \times \cdots \times\right.$ $\left.S_{i_{n}} \mid \exists \sigma \in W\left(\forall j=1 . . n\left(\sigma_{i_{j}}=f_{i_{j}}\right)\right)\right\}$. Then, the right conjunction expresses condition $A$, that, for any pair $i, j$ of mutually independent $\exists$ loise players, the projection $p_{\{i, j\}}(W)$ is a cartesian product (say, $\left.B^{i} \times B^{j}\right)$.

We immediately show with a counterexample that $\Omega_{\varphi}$ does not capture correctly the notion of "lack of knowledge of some player's strategy".

Counterexample 1 Consider the $C S$ sentence $\varphi=\exists x\left(\exists y / \emptyset_{-}\{x\}\right)(x \neq y)$. The intended meaning of it is that the player who picks a value for $y$ can see the value picked for $x$, but does not know the strategy used by the $\exists x$ player. With this interpretation, this sentence is obviously true in any structure with at least two elements (just let $\exists y$ choose $\beta(x)$, where $\beta$ is any bijection without fixed points). But $\Omega_{\varphi}$ does not entail this conclusion. To fix ideas, consider a two-element domain $M=\{a, b\}$. Notice that both strategies $f_{x}()=a, f_{y}(x)=b$ and $f_{x}()=b, f_{y}(x)=a$ are winning strategies for the $\exists$ loise team in the game of $M$ and $\varphi$. And yet, the strategy $f_{x}()=a, f_{y}(x)=a$ is not winning; thus, the "cartesian" constraint stated in $\Omega_{\varphi}$ is not respected.

So, the mere fact of being part of a uniform winning strategy does not work well as a notion of being a "best" strategy function in the present context. We will rather try with what seemed to emerge, in the previous sections, as the most reasonable notion of "best" strategy: dominance solvability. Another option could be to consider the set of winning equilibria: in that case we would obtain the solution concept known as (Luce and Raiffa's) solution in the strict sense (Luce and Raiffa (1957)). We will not pursue here this idea, but see Schelling (1960), Appendix C for a discussion of how this solution concept relates to issues of cooperation and signaling. The solution concept we develop can be seen instead as special case of Luce and Raiffa's solution in the complete weak sense $e^{14}$.

Let $D S_{\varphi}\left(f_{1}, \ldots, f_{m}\right)$ be the assertion that each of the strategy functions in the tuple $\left(f_{1}, \ldots, f_{m}\right)$ survives the process of iterated elimination of all weakly dominated strategy functions (as in the treatment of the semantics $\models_{D S}$ ). Let $\operatorname{DSWin}_{\varphi}\left(f_{1}, \ldots, f_{m}\right)$ be an abbreviation for the formula $D S_{\varphi}\left(f_{1}, \ldots, f_{m}\right) \wedge$

\footnotetext{
14 Actually, Luce and Raiffa argue that using, in this context, single-stage elimination of weakly dominated strategies instead of iterated elimination leads to a more plausible solution concept (what they call solution in the weak sense). This point is not investigated further in the present paper, but surely deserves future attention.
} 
$W_{\operatorname{Strat}}\left(f_{1}, \ldots, f_{m}\right)$. Finally, let $\Omega_{\varphi}^{C S}$ stand for the statement which one obtains from $\Omega_{\varphi}$ by replacing all occurrences of $\operatorname{WStrat}_{\varphi}$ with $\operatorname{DSWin}_{\varphi}$ :

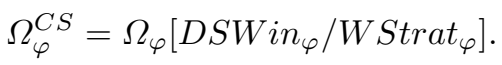

We then define, for any structure $M$ and $C S$ sentence $\varphi$ :

$$
M \vDash C S \varphi \Leftrightarrow \Omega_{\varphi}^{C S}
$$

This definition seems to work better, at least on the previous example.

Example 8 Consider again sentence $\varphi=\exists x\left(\exists y / \emptyset_{-}\{x\}\right)(x \neq y)$ and a twoelement structure $M$. The only strategies of $\exists$ loise that survive iterated elimination are $f_{x}()=a, f_{y}(x)=\operatorname{switch}(x)$ and $f_{x}^{\prime}()=b, f_{y}(x)=\operatorname{switch}(x)$, where $\operatorname{switch}(x)$ is once more the function that picks $b$ if $x$ equals $a$, and vice versa. The set $\left\{\left(f_{x}, f_{y}\right),\left(f_{x}^{\prime}, f_{y}\right)\right\}$ forms a cartesian product $\left\{f_{x}, f_{x}^{\prime}\right\} \times\left\{f_{y}\right\}$, and both strategies are winning; so, $M \models_{C S} \exists x\left(\exists y / \emptyset_{-}\{x\}\right)(x \neq y)$.

Example 9 This example shows that underscore sets allow disabling signalling possibilities. Let $\varphi=\forall x \exists z\left(\exists y /\{x\}_{-}\{z\}\right)(x=y)$, (here player $\exists y$ "does not know" the value chosen for $x$ and the strategy applied by $\exists z)$ and $M$ a twoelement structure as above. The strategy functions that survive the elimination process are $f_{z}(x)=x, f_{y}(z)=z, f_{z}^{\prime}(x)=\operatorname{switch}(x), f_{y}^{\prime}(z)=\operatorname{switch}(z)$. So, the surviving profiles are $\left(f_{z}, f_{y}\right),\left(f_{z}^{\prime}, f_{y}\right),\left(f_{z}, f_{y}^{\prime}\right),\left(f_{z}^{\prime}, f_{y}^{\prime}\right)$. Yet, both $\left(f_{z}, f_{y}\right)$ and $\left(f_{z}^{\prime}, f_{y}^{\prime}\right)$ are winning, but $\left(f_{z}, f_{y}^{\prime}\right)$ is not: so, the the set of winning strategies in the dominance solvability set is not a cartesian product. Thus, $M \forall_{C S} \varphi$. This is in accordance with the intuitive idea that, if the two $\exists$ loise players do not share a convention on how signals must be interpreted, then they cannot appropriately communicate.

Notice that this semantics does not coincide with $I F_{D S}$. In $I F_{D S}$ we required all the surviving strategies to be winning; here we require only that, among the strategies that survive, the winning ones satisfy conditions $A$ and $B$. But, viceversa, notice that the dominance solvability set itself (the set of strategies that survive the iterated elimination of weakly dominated strategies) always satisfies automatically condition $B$, and, on finite structures, also condition $A$. Asserting that a sentence is true according to $I F_{D S}$ amounts to saying that all strategies in the dominance solvability set are winning, so that we get:

Theorem 3 Let $M$ be a finite structure. Let $\varphi$ be an IF sentence, and $\varphi^{\prime}$ be obtained by adding arbitrary underscore sets to $\varphi$. Then

$$
M \models{ }_{D S} \varphi \Rightarrow M \models{ }_{C S} \varphi^{\prime} .
$$

Till now, we have not specified explicitly what the formula $D S_{\varphi}\left(f_{1}, \ldots, f_{n}\right)$ should be. Specifying that a strategy function $f_{i}$ is rational amounts to a $\Pi_{2}^{1}$ sentence:

$$
R A T_{i}: \forall g_{i}\left[\exists \pi\left(u_{\exists}\left(\pi_{-i}, g_{i}\right)<u_{\exists}\left(\pi_{-i}, f_{i}\right)\right) \vee \forall \pi\left(u_{\exists}\left(\pi_{-i}, g_{i}\right) \leq u_{\exists}\left(\pi_{-i}, f_{i}\right)\right)\right]
$$


where $\pi$ is a sequence of function symbols, and $u_{\exists}\left(\pi_{-i}, g_{i}\right) \leq u_{\exists}\left(\pi_{-i}, f_{i}\right)$ stands for

$$
\operatorname{Sk} \varphi\left[\pi_{-i} / h_{-i}, g_{i} / h_{i}\right] \rightarrow \operatorname{Sk} \varphi\left[\pi_{-i} / h_{-i}, f_{i} / h_{i}\right]
$$

and so on (here of course, writing $\pi_{-i} / h_{-i}$ we mean simultaneous substitution of each function variable in the sequence $\pi_{-i}$ for the corresponding function symbol from the sequence $h_{-i}$ ). Specifying that a strategy survives the second stage of elimination can be done by an identical formula, restricting all second-order quantifiers to the set of rational strategies. A careful process of extraction of quantifiers shows that this is (equivalent to) a $\Pi_{3}^{1}$ sentence. Analogously, the 3rd stage of elimination is expressed by a $\Pi_{5}^{1}$ sentence, and so on, in increasing complexity. Furthermore, none of these sentences captures dominance solvability, since the index of the final stage of elimination is bounded only by a function of the domain size $\left(O\left(2^{\operatorname{card}(M)}\right)\right)$; the statement " $f_{i}$ is in the dominance solvability set" is a fixed point of this sequence of sentences; and $D S_{\varphi}\left(f_{1}, \ldots, f_{n}\right)$ is a conjunction of these kinds of statements. So, the only upper bound we can see for expressivity of cartesian semantics is second-order fixpoint logic. We consider lower bounds in the next section.

We conclude this section noticing that in the literature there are at least two other examples of semantics of imperfect information which are based on cartesian products. One is Independence logic, on which we shall dwell later. The other is the definition of branching quantification given by G. Sher (Sher (1990)), which requires, for the truth of $B r\left(Q_{1}, Q_{2}\right) x y \psi$, that the set $S$ of tuples satisfying $\psi$ contains a maximal cartesian product $A \times B$ such that $A \in Q_{1}$ and $B \in Q_{2}$. Our condition seems to be more restrictive: we require a specific set (the winning subset of the dominance solvability set) to be a cartesian product.

If we identify each $I F$ quantifier $(\exists v / V)$ with the $C S$ quantifier $\left(\exists v / V \_\emptyset\right)$, we can translate each $I F$ sentence $\varphi$ into a $C S$ sentence $\varphi_{-}$; this translation preserves truth and, on finite structures, also non-truth. This holds because, when all underscore sets of a $C S$ sentence are empty, the rightmost clause of $\Omega_{\varphi}^{C S}$ is an empty conjunction (i.e., some valid sentence); while the leftmost conjunct of $\Omega_{\varphi}^{C S}$ just states that some strategy survives the iterated elimination procedure (which is equivalent, on finite structures, to the existence of a winning strategy - which is the usual truth condition of standard $I F$ semantics). So, $I F$ logic can be identified with a fragment of $C S$ :

Theorem 4 On finite structures, for any IF sentence $\varphi$,

$$
M \models \varphi \text { iff } M \models C S \varphi \_\emptyset \text {. }
$$

In the same spirit, a number of different semantics can be defined for $I F$ sentences depending on what kind of identification is made of the $I F$ formulas with (a set of) $C S$ formulas. Our favourite interpretation is the following:

Definition 16 Given an $I F$ formula $\psi$, we call $\psi^{C S}$ the $C S$ formula obtained replacing each quantifier $(\exists v / V)$ occurring in $\psi$ with the quantifier $\left(\exists v / V_{-} V^{\exists}\right)$, 
where $V^{\exists}$ is the subset of $V$ containing all the existentially quantified variables $^{15}$.

Then, for any $I F$ sentence $\varphi$, define: $M \models_{I F_{C S}} \varphi$ if $M \models_{C S} \varphi^{C S}$.

This makes slash sets forbid communication of both the kinds of information that we identified at the beginning of the paper (information about choices and information about individual strategies).

Definition 17 Given an $I F$ formula $\psi$, we call $\psi^{0}$ the $C S$ formula obtained replacing each quantifier $(\exists v / V)$ occurring in $\psi$ subordinated to existential quantifications over the variables in $W_{v}$ with the quantifier $\left(\exists v / V_{-} W_{v}\right)$.

Then, for any $I F$ sentence $\varphi$, define: $M \models_{0} \varphi$ if $M \models_{C S} \varphi^{0}$.

This corresponds to forbidding all exchange of information about individual strategies.

With these conventions, Theorem 3 can be specialized as follows:

Theorem 5 Let $\varphi$ be an IF sentence. Then, for any finite structure $M$,

$$
M \models{ }_{D S} \varphi \Rightarrow M=_{0} \varphi \Rightarrow M \models_{I F_{C S}} \varphi
$$

We must point out the peculiar role played, in relation to the $I F_{C S}$ interpretation, by the fragment $I F_{A R}^{\exists}$ of $I F$ sentences with action recall (for $\exists$ loise). These are the regular ${ }^{16}$ sentences that correspond to games in which ᄏloise never forgets her own moves; that is, sentences in which the slash sets of existential quantifiers do not contain existentially quantified variables (see Mann et al. (2011), 6.4). Now, the $I F_{C S}$ interpretation identifies each $I F_{A R}^{\exists}$ quantifier $(\exists v / V)$ with the $C S$ quantifier $\left(\exists v / V \_\emptyset\right)$, as the translation $\varphi \mapsto \varphi_{-} \emptyset$ did for the whole $I F$ logic; arguments similar to earlier ones show that, for $\varphi \in I F_{A R}^{\exists}$ and on finite structures, $M=\varphi$ iff $M \models_{I F_{C S}} \varphi^{C S}$. And $I F_{A R}^{\exists}$ is a proper extension of $F O L$; actually, it is as expressive as full $I F$ logic (Barbero et al. (2017)). Therefore, $I F_{C S}$ is at least as expressive as existential second-order logic.

And, summarizing the observations above, we have:

Theorem 6 1) Over finite structures, $C S$ is a conservative extension of $I F_{A R}^{\exists}$ (more precisely, of the image of $I F_{A R}^{\exists}$ under the $I F_{C S}$ translation).

2) $F O L<I F_{A R}^{\exists} \equiv I F \leq_{f i n} I F_{C S} \leq C S$.

On infinite structures, instead, $C S$ semantics, in its current form, suffers of the same problems that were pointed out for $I F_{R}$ and $I F_{D S}$ with regards to example 4 ; it is itself inadequate for providing a semantics for statements about infinite structures.

\footnotetext{
15 In case some variable $x$ from $V$ is quantified more than once above $(\exists v / V$ ) (as may happen in an irregular sentence), we consider it existentially quantified if the quantifier over $x$ which is closest above $(\exists v / V)$ is existential.

16 The regular sentences of $I F$ logic are those in which variables are not requantified; for example, $\forall x \exists x \psi$ is not regular. Sentence $\forall x P(x) \vee \exists x Q(x)$ instead is regular, because the two quantifiers over $x$ do not occur in the same branch of the synctactical tree (but it is not a strongly regular formula, because it contains two quantifiers over the same variable).
} 
Example 10 It is probably better, at this point, to check that the semantics introduced in the last sections do not falsify the critical sample sentence $\exists x(\exists y /\{x\}) x=0$ on $M=\left\{0^{M}, b\right\}$. The individual strategies for each player are the constant strategy 0 that picks $0^{M}$ and the constant strategy $b$ that picks $b$. In the first round of elimination of weakly dominated strategies, the strategy $b$ is eliminated from the set of strategies of player $\exists x$, and a fixed point of the elimination procedure is reached. Two possible profiles are left: $(0,0)$ and $(0, b)$. Since they are both winning, we have $M, \models_{R} \exists x(\exists y /\{x\}) x=0$ and $M, \mid{ }_{D S} \exists x(\exists y /\{x\}) x=0$. Since $\{(0,0),(0, b)\}=\{0\} \times\{0, b\}$ is a cartesian product, we also have that $M \models_{I F_{C S}} \exists x(\exists y /\{x\}) x=0$. The same argument also shows that $M \models_{0} \exists x(\exists y /\{x\}) x=0$.

\section{$10 \mathrm{CS}$ and independence atoms}

As was mentioned above, there is some formal analogy between our cartesian semantics and the Independence logic of Grädel and Väänänen (2013). This is one of many logics based on teams (sets of assignments with a common variable domain) that were proposed in the last decade. Team semantics was introduced in Hodges (1997) in order to give a compositional semantics to IF logic; since it makes no sense to speak of independence while describing a single state of affairs, satisfaction of open formulas needs to be defined on sets of assignments. The semantic clauses for first-order operators are just very explicit forms of the clauses of Tarskian semantics; but the framework is sufficiently general to allow defining satisfiability for $I F$ quantifiers. Given a team $X$ and a structure $M$, a function $F: X \rightarrow M$ is said to be $V$-uniform if $F(s)=F(t)$ whenever $s \sim_{V} t$. Then:

$$
M, X \models(\exists v / V) \psi \Leftrightarrow \exists F: X \rightarrow M, V \text {-uniform, s.t. } M, X[F / v] \models \psi
$$

where $X[F / v]$ is the team $\{s(F(s) / v) \mid s \in X\}$.

Similarly, in Independence logic one considers atomic formulas $\bar{x} \perp_{\bar{z}} \bar{y}$ $(\bar{x}, \bar{z}, \bar{y}$ being sequences of variables) with the special meaning

$\forall s, s^{\prime} \in X\left[s(\bar{z})=s^{\prime}(\bar{z}) \rightarrow \exists s^{\prime \prime} \in X\left(s^{\prime \prime}(\bar{z})=s(\bar{z}) \wedge s^{\prime \prime}(\bar{x})=s(\bar{x}) \wedge s^{\prime \prime}(\bar{y})=s^{\prime}(\bar{y})\right)\right]$

which is a notion of mutual independence which is considered in database theory. (Here $s(\bar{z})=s^{\prime}(\bar{z})$ stands for the conjunction of the equalities $s\left(z_{i}\right)=$ $s^{\prime}\left(z_{i}\right)$ for each $z_{i} \in \bar{z}$, etc.). One can immediately see a strong formal analogy between this clause and the semantic clause of $C S$ logic, especially in the case $\bar{z}=\langle\rangle$ (the empty sequence of variables).

In order to compare these two logics, we need to fix a translation between Independence formulas and $I F$ formulas. It was suggested in Grädel and Väänänen (2013) (and it seems to be the most natural choice) to replace each existential subformula of an IF formula

$$
(\exists y /\{\bar{x}\}) \psi(\bar{x}, y, \bar{z}),
$$


occurring inside the scope of quantifications over variables $\bar{x}, \bar{z}$, with the Independence formula

$$
\exists y\left(y \perp_{\bar{z}} \bar{x} \wedge \psi\right) .
$$

(An inverse translation is not obvious; first of all, because it is not clear how to preserve the parameter $\bar{z}$, and secondly, because slash sets are in a bijection with quantifications, while the occurrences of independence atoms may be more numerous than the quantifications).

Using this interpretation we can see that, despite the formal analogies, $C S$ and Independence logic are radically different systems; Independence logic is not a logic inhibiting cooperation, as the following example shows.

Example 11 The translation of sentence $\exists x(\exists y /\{x\})(x=y)$ into Independence logic is $\exists x(x \perp\langle\rangle \wedge \exists y(y \perp x \wedge x=y))$, where again we denote by \langle\rangle the empty sequence of variables. The satisfaction clause for $x \perp\langle\rangle$ is trivial, so this sentence is equivalent to $\exists x \exists y(y \perp x \wedge x=y)$. An Independence sentence is said to be true if it is satisfied by team $\{\emptyset\}$. Then

$$
\begin{gathered}
M,\{\emptyset\} \models \exists x \exists y(y \perp x \wedge x=y) \\
\mathbb{\Downarrow} \\
\exists C:\{\emptyset\} \rightarrow M(M,\{\emptyset\}[C / x] \models \exists y(y \perp x \wedge x=y)) \\
\mathbb{\Downarrow} \\
\exists C:\{\emptyset\} \rightarrow M, \exists F:\{\emptyset\}[C / x] \rightarrow M(M,\{\emptyset\}[C / x][F / y] \models(y \perp x \wedge x=y)) \\
\mathbb{\Downarrow} \\
\exists C, F \ldots\left(\forall s \in X(s(x)=s(y)) \wedge \forall s, s^{\prime} \in X \exists s^{\prime \prime} \in X\left(s^{\prime \prime}(x)=s(x) \wedge s^{\prime \prime}(y)=s^{\prime}(y)\right)\right),
\end{gathered}
$$

where in the last line $X$ stands for $\{\emptyset\}[C / x][F / y]$.

Choosing for $C$ any constant $c \in M$, and for $F$ the "identity function" $s \mapsto$ $s(x)$, we have $X=\{\{\langle x, c\rangle,\langle y, c\rangle\}\}$, a team containing a single assignment. One can easily see that $X$ satisfies the two conjuncts in the last formula, so that $M=\exists x \exists y(y \perp x \wedge x=y)$.

The difference between the two logics is that in $I F_{C S}$ we impose the cartesian product condition on global strategies, while in Independence logic the condition is applied only to some specific set of assignments, which is determined by the form of the team which is constructed (by means of particular choices) while evaluating compositionally the fomula. 


\section{$11 C S, S G S$ and the rest: some comparative examples}

Notice at this point that the system $I F_{C S}$ does not coincide with Subgame Semantics. Indeed, Hodges' signalling formula

$$
\forall x \exists z(\exists y /\{x\})(x=y)
$$

is not true on $M=\{a, b\}$ according to Subgame Semantics; it is true in $I F_{C S}$. Indeed, since this formula does not contain any declaration of independence between existential quantifiers, its truth condition coincides with the standard truth condition of $I F$ logic, and (5) is known to be true in $I F$ logic.

Notice, instead, that formula (1) (section 3) is false in $I F_{C S}$ : no strategy function is weakly dominated, so that the "cartesian product" condition must be imposed on the whole set of winning strategies. But the only winning choices (for $x$ and $y$, respectively) are $(a, a)$ and $(b, b) ;(a, b)$ is not a winning strategy, and so the set of winning strategies does not form a cartesian product.

So, we have some evidence that $I F_{C S}$ could be a system which 1 . prevents forbidden coordination of strategies, but at the same time 2. allows signalling.

Is it possible, instead, to particularize $C S$ logic in order to obtain a system which, in the same fashion of subgame semantics, forbids both signalling and unwanted cooperation? A good candidate for this role could be the semantics $I F_{0}$ which we introduced earlier (although, this may seem to be an exaggerate solution, since it means forbidding all forms of cooperation, and not only those which are unwanted).

So, does this semantics really coincide with subgame semantics? A theoretical comparison seems to be difficult. We shall look at some enlightening examples.

Example 12 $\left(I F_{0} \nsubseteq I F_{R}, I F_{R} \subset I F_{D S}\right)$

Let $M=\left\{a_{1}, a_{2}, a_{3}\right\}$ be a set of three distinct objects. We want to describe a strategic game of three players $\exists x, \exists y, \forall z$ (each of them having three constant strategies $\left.a_{1}, a_{2}, a_{3}\right)$. Consider the following table. Rows correspond to strategies of $\exists x$, columns to strategies of $\exists y$. The three numbers in each cell, from left to right, describe the utility function of the $\exists$ loise players in case $\forall$ belard answers to their pair of strategy functions by choosing $a_{1}, a_{2}$ or $a_{3}$ respectively for $z$.

\begin{tabular}{|l|c|c|c|}
\hline & $a_{1}$ & $a_{2}$ & $a_{3}$ \\
\hline$a_{1}$ & $(0,0,1)$ & $(1,1,1)$ & $(1,1,1)$ \\
\hline$a_{2}$ & $(0,0,1)$ & $(1,1,1)$ & $(1,1,1)$ \\
\hline$a_{3}$ & $(0,1,1)$ & $(0,1,1)$ & $(0,1,1)$ \\
\hline
\end{tabular}

Now define $S^{M}(x, y, z)$ to be true if and only if the $z$-th number of the sequence contained in the box corresponding to row $a_{x}$ and column $a_{y}$ is a 1. Let $\mathbb{M}=\left(M, S^{M}\right)$. The IF formula $\zeta=\forall z(\exists x /\{z\})(\exists y /\{x, z\}) S(x, y, z)$, evaluated on $\mathbb{M}$, defines the game described in the table.

Looking at the table, we see that there are four uniform strategies for team $\exists$ loise which survive iterated elimination of weakly dominated strategies: $\left(a_{1}, a_{2}\right),\left(a_{1}, a_{3}\right),\left(a_{2}, a_{2}\right),\left(a_{2}, a_{3}\right)$. For $\forall$ belard, only strategy $a_{1}$ survives. 


\begin{tabular}{|c|c|c|}
\hline & $a_{2}$ & $a_{3}$ \\
\hline$a_{1}$ & $a_{1}: 1$ & 1 \\
\hline$a_{2}$ & 1 & 1 \\
\hline
\end{tabular}

The surviving profiles are all winning for $\exists$ loise, and they form a cartesian product. So,

$$
\begin{gathered}
\mathbb{M} \models_{0} \forall z(\exists x /\{z\})(\exists y /\{x, z\}) S(x, y, z) \\
\mathbb{M} \models_{D S} \forall z(\exists x /\{z\})(\exists y /\{x, z\}) S(x, y, z) .
\end{gathered}
$$

But if we perform only one round of elimination of weakly dominated strategies, we obtain (since $u_{\exists}\left(a_{3}, a_{1}, a_{2}\right)=1>0=u_{\exists}\left(a_{2}, a_{1}, a_{2}\right)$ ) the table:

\begin{tabular}{|c|c|c|}
\hline & $a_{2}$ & $a_{3}$ \\
\hline$a_{1}$ & $a_{1}: 1$ & 1 \\
\hline$a_{2}$ & 1 & 1 \\
\hline$a_{3}$ & 0 & 0 \\
\hline
\end{tabular}

So, there are some profiles, like the triple of strategies $\left(a_{3}, a_{3}, a_{1}\right)$ for $\exists x, \exists y, \forall z$, which are formed by rational strategy functions, but they are a loss for $\exists$ loise. (We may also state it like this: $\left(a_{3}, a_{3}\right)$ is not a winning strategy for $\exists$ loise team, since it loses against $\forall z$ choosing $\left.a_{1}\right)$.

So, $\mathbb{M} \nvdash_{R} \forall z(\exists x /\{z\})(\exists y /\{x, z\}) S(x, y, z)$. We may conclude that $I F_{R}$ is strictly included in $I F_{D S}$ and does not coincide with our minimal logic with cartesian semantics, $I F_{0}$ (not even on finite domains).

\section{Example $13\left(I F_{S G S^{2}} \nsubseteq I F_{R}\right)$}

What does Subgame Semantics say about the last sentence? Our first impression was that it should be untrue, as in $I F_{R}$. But, if you draw the game tree, you can see that there is one strategy for $\exists$ loise team ("choose $a_{2}$ for $x$ and also for $y ")$ which, applied in any node of the second stage of the game, brings to a winning position, with one exception: node $\left(\left(z, a_{1}\right),\left(x, a_{3}\right)\right)$. But this node is a losing position - whatever choice $\exists$ loise makes for $y$ at this point leads to a loss - and so it is not required that the strategy leads to a winning position from there. Thus $\mathbb{M} \models_{S G S^{2}} \forall z(\exists x /\{z\})(\exists y /\{x, z\}) S(x, y, z)$. So, we see that, even on finite structures, $I F_{R}$ is not the correct game-theoretical justification of Subgame Semantics, in spite of the similarity pointed out by Janssen (section 3 of Janssen (2005), section 5 of Janssen (2007)). We may suspect that $I F_{0}$ is not, either; the following example will prove this fact.

Example $14\left(I F_{0} \nsubseteq I F_{S G S^{2}}, I F_{C S} \nsubseteq I F_{S G S^{2}}, I F_{D S} \subset_{f i n} I F_{C S}, I F_{D S} \subset_{f i n}\right.$ $I F_{0}$ ) Consider again the sentence $\zeta$ of the previous examples, on a structure $\mathbb{M}^{\prime}$ with relation $S^{M}$ defined by the following table:

\begin{tabular}{|c|c|c|c|}
\hline & $a_{1}$ & $a_{2}$ & $a_{3}$ \\
\hline$a_{1}$ & $(0,0,1)$ & $(1,1,1)$ & $(1,1,1)$ \\
\hline$a_{2}$ & $(0,0,1)$ & $(1,1,1)$ & $(1,1,1)$ \\
\hline$a_{3}$ & $(1,1,0)$ & $(0,1,1)$ & $(0,1,1)$ \\
\hline
\end{tabular}


Here, no individual strategy is weakly dominated. But not all pairs of strategies of the $\exists$ loise players are winning; so, $\mathbb{M}^{\prime} \not \neq_{D S} \zeta$ and $\mathbb{M}^{\prime} \not_{R} \zeta$. But the set of the winning pairs, $\left(a_{1}, a_{2}\right),\left(a_{1}, a_{3}\right),\left(a_{2}, a_{2}\right),\left(a_{2}, a_{3}\right)$, is a cartesian product; so $\mathbb{M}^{\prime}=_{I F_{0}} \zeta$, and also $\mathbb{M}^{\prime} \models_{I F_{C S}} \zeta$.

If instead one looks at each single winning strategy of the $\exists$ loise team, one can see that the strategy function for $\exists y$ is losing in the history which only differs in that $\exists x$ chooses $a_{3}$, and $\forall z$ chooses $a_{1}$; and none of the corresponding histories is a losing position. So, $\mathbb{M}^{\prime} \not \neq_{S G S^{2}} \zeta$.

By example 13 we know that $M \models_{S G S^{2}} \varphi$ does not imply $M=_{R} \varphi$, but a possibility remains open that, on finite structures, $I F_{R} \subset_{f i n} I F_{S G S^{2}}$.

Looking globally at the previous examples, we have the strict inclusions $I F_{R} \subset I F_{D S} \subset_{f i n} I F_{0} \subset I F_{C S}$ (the third of which holds not only because the examples above, but also because the two semantics disagree about the truth values of Hodges' signalling formula).

Comparing these logics with Subgame Semantics seems more difficult; we do not even know if $I F_{R}$ is contained in it. $I F_{0}$ (and thus $I F_{C S}$ ) might contain $I F_{S G S^{2}}$, but not be equal to it; we cannot instead exclude the coincidence of $I F_{D S}$ and $I F_{S G S^{2}}$ on finite structures, which would yield a partial gametheoretical justification of Subgame Semantics.

It seems difficult to compare these logics with the standard $I F$ semantics, or with (the expressive power of) first-order logic (we only know that $I F_{S G S^{2}} \equiv$ $F O$, and $\left.I F_{A R}^{\exists} \leq_{\text {fin }} I F_{C S}\right)$.

\section{Conclusions}

We analyzed the few papers in the literature where non-standard semantics for IF syntax are introduced which falsify (or, more precisely, not-verify) Janssen's example (1), and we added a few new proposals (including one semantics, $I F_{C S}$, which falsifies the example but preserves some signalling phenomena). We did not reach a definitive conclusion on which of these frameworks better grasps the epistemic view of imperfect information; for sure, some of these semantics have strong counterindications. That is the case of $I W D$, whose game-theoretical justification seems ill-founded; of $W D$, which makes false even some trivial first-order validity; of $I F_{S S S}$ and $I F_{S G S^{1}}$, which give totally counterintuitive truth values for some sentences. Yet, these last two logics may still have some interest, in that they seem to be able to characterize some non-elementary classes.

The semantics which survive this selection have been compared; $I F_{C S}$ differs from all the others because of its tolerance of signalling; some systems were proved to differ from each other $\left(I F_{0}, I F_{R}\right.$ and $\left.I F_{S G S^{2}}\right)$; the differentiation between the last two disproves a conjecture of Janssen, according to which the notion of rationalizability could be a game-theoretical account of Subgame Semantics on finite structures. The possibility is left open that $I F_{S G S^{2}}$ be a proper fragment of $I F_{0}$, or at least a proper fragment of $I F_{C S}$. Furthermore, 
we proved the proper inclusions $I F_{R} \subset I F_{D S} \subset_{f i n} I F_{0} \subset I F_{C S}$ (the second inclusion being correct only over finite structures). Many question are left open. One is: how to compare the expressive power of $I F_{0}$ and $I F_{C S}$ with that of standard $I F$ logic. The recent proof (Barbero et al. (2017)) of $I F_{A R}^{\exists} \equiv \Sigma_{1}^{1}$ gives a partial answer to this point: $I F \leq_{\text {fin }} I F_{C S}$. Another thing we wonder about is the status of semantics $I F_{D S}$. We excluded that it may coincide with $I F_{S G S^{2}}$, but the possibility is left open that it provides a correct justification for Subgame Semantics over finite structures (while $I F_{0}$ contains too many truths). Thirdly, we think that perhaps also the logic $C S$, which has a more general syntax, and which was here considered instrumentally for the study of IF logic, would deserve to be studied on its own. Fourth, we suspect that all of these systems might be compared more effectively if probabilistic extensions were developed for them (as it was the case for the standard IF semantics).

We wish to point out that the game-theoretical solution concept which underlies $C S$ semantics, and which we developed here with logical applications in mind, might as well be of interest for game theory itself. Indeed, the usual formal models of (either noncooperative or cooperative) games seems to be completely inadequate to describe correctly the epistemic relationships among players in conditions of imperfect recall; this inadequacy seems to amount to the lack of simple instruments, in the classical model, for the description of knowledge about strategies. A solution concept like that underlying $C S$ semantics can be used to account for this missing possibility.

\section{A bibliographical note}

At the time of the final redaction of this article, we were informed of the existence of a paper of J. van Benthem (van Benthem (2004)) that predates the publications described in section 7 , and which anticipates the idea of using elimination of strategies in the evaluation of $I F$ sentences. The influence of this work on the above-mentioned publications might be confirmed by the presence of a bibliographical reference, in Sevenster (2007), to an earlier draft of the book Logic in Games (van Benthem (2014)), in which the arguments of van Benthem (2004) are briefly reviewed.

\section{Acknowledgements}

This paper was completed under the Academy of Finland Grant n.286991.

Many thanks are due to the anonymous reviewers, whose suggestions were of great help in improving the readability of the paper and for correcting a few mistakes that were present in an earlier draft. 


\section{References}

Barbero, F. (2013). On existential declarations of independence in IF logic. The Review of Symbolic Logic, 6:254-280.

Barbero, F. (2014). Standard and nonstandard semantics for languages of imperfect information. PhD thesis, Universitá di Torino.

Barbero, F., Hella, L., and Rönnholm, R. (2017). Independence-Friendly logic without Henkin quantification. To appear in the LNCS Proceedings of WoLLIC 2017.

van Benthem, J. (2004). Probabilistic features in logic games. In Symons, J. and Kolak, D., editors, Quantifiers, Questions and Quantum Physics. Essays on the Philosophy of Jaakko Hintikka, pages 189-195. Springer.

van Benthem, J. (2014). Logic in Games. MIT Press.

Cameron, P. and Hodges, W. (2001). Some combinatorics of imperfect information. Journal of Symbolic Logic, 66:673-684.

Enderton, H. B. (1970). Finite partially ordered quantifiers. Mathematical Logic Quarterly, 16(8):393-397.

Grädel, E. and Väänänen, J. (2013). Dependence and independence. Studia Logica, 101:399-410.

Halpern, J. (1996). On ambiguities in the interpretation of game trees. Games and Economic Behavior, 20:66-96.

Halpern, J. and Pass, R. (2009). A logical characterization of iterated admissibility. In Heifetz, A., editor, TARK '09 Proceedings of the 12th Conference on Theoretical Aspects of Rationality and Knowledge, pages 146-155. ACM New York, NY, USA.

Henkin, L. (1961). Some remarks on infinitely long formulas. In Infinitistic methods, pages 167-183, Oxford-London-New York-Paris. Pergamon Press.

Hintikka, J. (1996). The Principles of Mathematics Revisited. Cambridge University Press, Cambridge, UK.

Hintikka, J. (2013). Function logic and the theory of computability. APA Newsletter on Philosophy and Computers, 13:10-19.

Hintikka, J. and Sandu, G. (1989). Informational independence as a semantical phenomenon. In Fenstad et al, J. E., editor, Logic, Methodology and Philosophy of Science VIII, pages 571-589. Elsevier Science Publishers B.V.

Hodges, W. (1997). Compositional semantics for a language of imperfect information. Logic Journal of the IGPL, 5:539-563.

Hodges, W. (2012). Note on janssen's 'Independent choices and the interpretation of IF logic'. Working paper.

Humberstone, L. (1987). Critical notice of J. Hintikka and J. Kulas, The game of language. Mind, 96:99-107.

Jamroga, W. and van der Hoek, W. (2004). Agents that know how to play. Fundamenta Informaticae, 63:185-219.

Janssen, T. M. V. (2001). On the definition of independence in logic. Technical report, Workshop on Logic and Games, Helsinki.

Janssen, T. M. V. (2002). Independent choices and the interpretation of IF logic. Journal of Logic, Language and Information, 11:367-387. 
Janssen, T. M. V. (2005). Independence friendly logic as a strategic game. In Dekker, P. and Franke, M., editors, Proceedings Fifteenth Amsterdam Colloquium, pages 125-130.

Janssen, T. M. V. (2007). Independence and Hintikka games. Technical report, ILLC research report and technical notes series.

Luce, R. D. and Raiffa, H. (1957). Games and decisions: introduction and critical survey. New York: Wiley.

Mann, A. L., Sandu, G., and Sevenster, M. (2011). Independence-Friendly Logic - a Game-Theoretic Approach, volume 386 of London Mathematical Society lecture note series. Cambridge University Press.

Moulin, H. (1979). Dominance solvable voting schemes. Econometrica, 47:1337-1351.

Osborne, M. J. and Rubinstein, A. (1994). A course in game theory. MIT Press, Cambridge.

Pearce, D. G. (1984). Rationalizable strategic behavior and the problem of perfection. Econometrica, 52:1029-1050.

Sandu, G. (1993). On the logic of informational independence and its applications. Journal of Philosophical Logic, 22:29-60.

Schelling, T. C. (1960). The Strategy of Conflict. Harvard University Press, Cambridge.

Sevenster, M. (2007). A strategic perspective on if games. Technical report, ILLC scientific publications, Inst. for Logic, Language and Computation.

Sevenster, M. (2014). Dichotomy result for independence-friendly prefixes of generalized quantifiers. The Journal of Symbolic Logic, 79(04):1224-1246.

Sher, G. (1990). Ways of branching quantifiers. Linguistics and Philosophy, 13:393-422.

Väänänen, J. (2007). Dependence Logic: A New Approach to Independence Friendly Logic, volume 70 of London Mathematical Society Student Texts. Cambridge University Press. 